\title{
ON THE EXCURSION RANDOM MEASURE OF STATIONARY PROCESSES ${ }^{1}$
}

By TAILEN Hsing and M. R. LeAdBetter

\author{
Texas A\&M University and University of North Carolina
}

\begin{abstract}
The excursion random measure $\zeta$ of a stationary process is defined on sets $E \subset(-\infty, \infty) \times(0, \infty)$, as the time which the process (suitably normalized) spends in the set $E$. Particular cases thus include a multitude of features (including sojourn times) related to high levels. It is therefore not surprising that a single limit theorem for $\zeta$ at high levels contains a wide variety of useful extremal and high level exceedance results for the stationary process itself.

The theory given for the excursion random measure demonstrates, under very general conditions, its asymptotic infinite divisibility with certain stability and independence of increments properties leading to its asymptotic distribution (Theorem 4.1). The results are illustrated by a number of examples including stable and Gaussian processes.
\end{abstract}

1. Introduction. Random measure theory has important applications in the study of extremes. As usual, extremes refer to the very large and small observations in a large sample. In this paper, however, we focus on the right extremes since extensions to include extremes on both directions are straightforward. Cramér and Leadbetter (1967), Leadbetter, Lindgren and Rootzén (1983), Resnick (1987) and Leadbetter and Rootzén (1988) illustrate the substantial benefit of proving functional limit theorems for point processes recording typically either the high-level exceedance positions by a random sequence (with discrete-time space), or high-level upcrossing positions of a random process (with continuous-time space). To motivate the problems considered in this paper, we now review one such approach in the discrete-time case. Let $\left\{\xi_{j}\right\}$ be a strictly stationary sequence of random variables. Define the point process $\eta_{n}$ by

$$
\left.\eta_{n}(E)=\# j:\left(j / n, u_{n}^{-1}\left(\xi_{j}\right)\right) \in E\right\} \quad \text { for Borel sets } E \text { in } \Re_{+}^{2},
$$

where $\Re_{+}^{2}:=(-\infty, \infty) \times(0, \infty)$, and $u_{n}$ is some decreasing function on $\Re_{+}:=$ $(0, \infty)$ which can typically be taken to satisfy $P\left[\xi_{1}>u_{n}(\tau)\right] \sim \tau / n$ as $n \rightarrow \infty$, $\tau>0$. While other normalizations can be used, we feel that this particular one leads to the simplest "self-similar" representation of the distributional limit. If $P\left\{\max _{1 \leq j \leq n} \xi_{j} \leq a_{n} x+b_{n}\right\} \rightarrow{ }_{w} G(x)$, then $u_{n}(\tau)$ can simply be $a_{n} G^{-1}\left(e^{-\tau}\right)+$ $b_{n}$. Note that the scalings of both time and space allow $\eta_{n}$ to capture the positions as well as the magnitudes of the extremes $\left\{\xi_{j}\right\}$. It is therefore natural

Received J anuary 1995; revised J anuary 1998.

${ }^{1}$ Supported by ONR Grants N00014-92-J -1007 and N00014-93-1-0043.

AMS 1991 subject classifications. 60F 05, 60G10.

Key words and phrases. Extremes, infinite divisibility, sojourns, weak convergence. 
that the weak convergence of $\eta_{n}$ is equivalent to the joint weak convergence of the extreme order statistics of $\xi_{1}, \ldots, \xi_{n}$. See Hsing (1987), Resnick (1987), O'Brien, Torfs and Vervaat (1990) and the references therein.

In this paper we consider the corresponding version of $\eta_{n}$ for continuoustime space stationary processes. Let $\left\{\xi_{t}, t \in \mathfrak{R}\right\}$ be a strictly stationary process defined on some probability space, where we make the minimal assumption that the process is jointly measurable in $t$ and $\omega$ (i.e., measurable with respect to the product $\sigma$-field of the Borel $\sigma$-field of $\Re$ and the $\sigma$-field of the probability space). Define the random measure $\zeta_{T}$ by

$\zeta_{T}(E)=$ Lebesgue measure of $\left\{t:\left(t / T, u_{T}^{-1}\left(\xi_{t}\right)\right) \in E\right\}$ for Borel sets $E$ in $\Re_{+}^{2}$, where $u_{T}$ is an appropriate normalization whose role is similar to that of $u_{n}$ in $\eta_{n}$. We call $\zeta_{T}$ (or $\left\{\zeta_{T}, T>0\right\}$ ) the excursion random measure of $\left\{\xi_{t}\right\}$. The goal of this paper is to consider issues related to the asymptotic distributional properties of $\zeta_{T}$.

Many features of the extremes of $\left\{\xi_{t}\right\}$ are recorded by $\zeta_{T}$ for large $T$. As an elementary illustration, for sets $E_{i}=A \times\left(0, \tau_{i}\right), 1 \leq i \leq k$, where $A \subset \Re_{+}$ and $\tau_{i}>0$, the random vector $\left(\zeta_{T}\left(E_{1}\right), \zeta_{T}\left(E_{2}\right), \ldots, \zeta_{T}\left(E_{k}\right)\right)$ records simultaneously the amount of time in $T \cdot A$ that the process $\left\{\xi_{t}\right\}$ spends in the "rare sets" $\left(u_{T}\left(\tau_{i}\right), \infty\right)$. Clearly, the time and spatial sets here can be much more general. Hence, with application of the continuous mapping theorem, the class of limit theorems that can be derived from the asymptotic distribution of $\zeta_{T}$ go beyond those in the literature on the sojourns in rare sets by stationary processes [cf. Berman (1980, 1982, 1983)]. Furthermore, this approach is rather general from the following viewpoints. First, since only the amount of excursion time is relevant in defining $\zeta_{T}$, very little is required in terms of sample path regularity. Furthermore, deriving the asymptotic distribution of $\zeta_{T}$ is usually relatively straightforward and, in particular, does not require the underlying process $\left\{\xi_{t}\right\}$ to satisfy a stringent mixing condition such as the strong mixing condition. Thus, the approach applies to a very general class of processes and can potentially lead to a wide range of applications.

A major difference between the continuous and discrete-time cases is that for the continuous-time case, in addition to the "global" normalization $u_{T}$, a "local" normalization on the durations of the high-level excursions is also required for the distribution of $\zeta_{T}$ to stabilize for large $T$. This normalization, which differs from process to process, is in the form of a multiplicative constant $a_{T}$ of $\zeta_{T}$. In some cases (for example, moving average stable processes) it is possible to take $a_{T}$ as a fixed constant. However, typically up- and downcrossings of a high level by $\left\{\xi_{t}\right\}$ occur at time scale smaller than that of the process, in which case $a_{T} \rightarrow \infty$ at a speed that reflects the "natural time scale" for observing high-level excursions. In the Gaussian case $a_{T}$ can be taken as $(\log T)^{1 / 2}$.

The main theme of this paper will be approached from a completely general point of view. First, in Section 2 we describe the theoretical foundation of the excursion random measure. There we also define a mixing condition under which we deduce a set of properties that must hold in the limit for 
the excursion random measure. The class of random measures possessing such properties is characterized in Section 3, extending the results in Mori (1977) and Hsing (1987). In Section 4, we develop an approach for deriving the asymptotic distribution of the excursion random measure in terms of the global and local properties of high-level excursions. In Section 5, four examples of different natures are given to illustrate the various aspects of the limiting excursion random measure. In particular, a moving average stable process and a Gaussian process are considered. For clarity of presentation, all the proofs are collected in Section 6.

In the remaining part of this section, we briefly review some basic concepts and terminology from random measure theory relevant to this paper. The reader is referred to Kallenberg (1983) and Matthes, Kerstan and Mecke (1978) for details. In this paper, a subspace of a topological space has the relative topology and a product space of topological spaces has the product topology. For a topological space $T, \sigma(T)$ denotes the Borel $\sigma$-field of $T$. The random measures in this paper will have state (or phase) space $S$ equal to either $\Re_{+}^{2}$, or $\Re_{+}$, which have the usual topologies. Also let $\mathscr{B}=\mathscr{B}(S)$ be the collection of all bounded (i.e., relatively compact) sets in $\sigma(S)$ and $\mathscr{T}=\mathscr{T}(S)$ the class of nonnegative measurable functions on $S$. Denote by $\mathscr{M}=\mathscr{M}(S)$ the collection of locally finite measures on $(S, \sigma(S))$. Here $\mathscr{M}$ is assumed to have the vague topology which generates the Borel $\sigma$-field $\sigma(\mathscr{M})$. The null measure in $\mathscr{M}$ is denoted by 0 when there is no risk of ambiguity. For $\mu \in \mathscr{M}$ and $f \in \mathscr{F}$, write $\mu f=\int_{S} f d \mu$. A random measure $\eta$ on $(S, \sigma(S))$ is a measurable mapping from some probability space $(\Omega, \mathscr{B}, P)$ to $(\mathscr{M}, \sigma(\mathscr{M}))$. The distribution of $\eta, P \circ \eta^{-1}$, is uniquely determined by its Laplace transform

$$
L_{\eta}(f)=\mathscr{E} \exp (-\eta f), \quad f \in \mathscr{F} .
$$

Then $\eta$ is infinitely divisible if, for each $n \geq 1$, there exist some i.i.d. random measures $\eta_{1}, \ldots, \eta_{n}$ such that $\eta$ has the same distribution as $\sum_{i=1}^{n} \eta_{i}$. The following result is fundamental.

THEOREM 1.1 [cf. Kallenberg (1983), Theorem 6.1]. The relation

$$
-\log \mathscr{E} e^{-\eta f}=\alpha f+\int_{\mathscr{M} \backslash\{0\}}\left(1-e^{-\mu f}\right) \lambda(d \mu), \quad f \in \mathscr{F},
$$

defines a unique correspondence between the distributions of all infinitely divisiblerandom measures $\eta$ on $(S, \sigma(S))$ and the class of all pairs $(\alpha, \lambda)$, where $\alpha$ is in $\mathscr{M}$ and $\lambda$ is a measure on $\mathscr{M} \backslash\{0\}$ satisfying

$$
\int_{\mathscr{M} \backslash\{0\}}\left(1-e^{-\mu B}\right) \lambda(d \mu)<\infty, \quad B \in \mathscr{B} .
$$

For simplicity of terminology, we shall write in this case $\eta_{d}:=\alpha$ and $\eta_{r}:=$ $\eta-\eta_{d}$.

2. The excursion random measure. Let $\left\{\xi_{t}, t \in \Re\right\}$ be a strictly stationary process that is jointly measurable in $t$ and $\omega$. Let $\left\{u_{T}, T>0\right\}$ be 
a collection of normalizing functions on $\Re_{+}=(0, \infty)$ with the following properties.

(U1) $u_{T}$ is continuous and strictly decreasing for each $T>0$;

(U2) $\lim _{T \rightarrow \infty} u_{\sigma T}^{-1} \circ u_{T}(\tau)=\sigma \tau$ for each $\sigma$ and $\tau>0$,

where $u_{T}^{-1}(y)=\sup \left\{\tau>0: u_{T}(\tau) \geq y\right\}$ and “०" stands for the composition of functions. Condition ( $U 1)$ is a smoothness condition, which is included here for convenience. Condition (U2) enables a cleaner description of the asymptotic distribution of the extremes. Condition (U2) is satisfied if, for example, $u_{T}$ satisfies (U 1) and

$$
\lim _{T \rightarrow \infty} \operatorname{TP}\left\{M(1)>u_{T}(\tau)\right\}=\tau, \quad \tau>0
$$

or

$$
\lim _{T \rightarrow \infty} P\left\{M(\sigma T) \leq u_{T}(\tau)\right\}=e^{-\sigma \tau}, \quad \sigma, \tau>0,
$$

where

$$
M(t)=\sup _{0 \leq s<t} \xi_{s}, \quad t>0 .
$$

Note that for a weakly dependent process, (2.1) and (2.2) are closely related conditions. If the discrete time process $\left\{X_{i}=\sup _{i \leq t<i+1} \xi_{t}\right\}$ has a nonzero extremal index $\theta$ [cf. Leadbetter (1983)], then (2.2) holds with $u_{T}(\tau)=v_{T}(\tau / \theta)$ whenever (2.1) holds with $u_{T}(\tau)=v_{T}(\tau)$ for some $v_{T}$. The role of $u_{T}$ is typically to estimate the quantiles of the extremes of $\xi_{t}, 0 \leq t<T$. Ther efore, the function $u_{T}$ satisfying (2.1) or (2.2) corresponds to the most useful normalization. For the most part, we will make that assumption. However, there is no need to confine the treatment to these two possibilities at first.

The excursion random measure $\zeta_{T}$ defined in Section 1 may clearly be expressed as

$$
\zeta_{T}(E)=\int_{\Re} I_{E}\left(t / T, u_{T}^{-1}\left(\xi_{t}\right)\right) d t, \quad E \in \sigma\left(\Re_{+}^{2}\right),
$$

where $I_{E}(x, y)=0$ or 1 according as $(x, y) \notin E$ or $(x, y) \in E$. It follows readily from the joint measurability of $\left\{\xi_{t}\right\}$ and Fubini's theorem that $\zeta_{T}(E)$ is measurable for every Borel set $E$ and hence $\zeta_{T}$ is a well-defined random measure [cf. Kallenberg (1983), Lemma 1.4].

As explained in Section 1, a multiplicative constant $a_{T}$ of $\zeta_{T}$ is generally necessary in order for the distribution of $\zeta_{T}$ to converge. Roughly speaking, $a_{T}^{-1}$ reflects the time scale of the high-level excursions. Therefore, it is desirable that $a_{T}$ and $u_{T}$ be such that $a_{T}$ times the mean excursion time of $\xi_{t}$ above $u_{T}(\tau)$ for $t \in[0, T]$ is bounded away from 0 and $\infty$; that is,

$$
0<\liminf _{T \rightarrow \infty} a_{T} T P\left\{\xi_{1}>u_{T}(\tau)\right\} \leq \limsup _{T \rightarrow \infty} a_{T} T P\left\{\xi_{1}>u_{T}(\tau)\right\}<\infty .
$$


If additionally $\operatorname{TP}\left\{M(1)>u_{T}(\tau)\right\}$ is also bounded away from 0 and $\infty$ [e.g., under (2.1)], then

$$
a_{T} \asymp \frac{T P\left\{M(1)>u_{T}(\tau)\right\}}{T P\left\{\xi_{1}>u_{T}(\tau)\right\}} \frac{1}{T P\left\{M(1)>u_{T}(\tau)\right\}} \asymp \frac{P\left\{M(1)>u_{T}(\tau)\right\}}{P\left\{\xi_{1}>u_{T}(\tau)\right\}},
$$

in which case $a_{T}$ is bounded away from 0 . We will follow this up in Remark 1 after Proposition 2.1.

One of the major purposes of this paper is to consider the possible asymptotic distributions of the excursion random measure for weakly dependent $\left\{\xi_{t}\right\}$. In particular we are interested in the weak dependence defined by the following mixing condition. For $0<s<t<T$ and $\tau_{i}>0,1 \leq i \leq m$, write

$$
\mathscr{B}_{s, t}^{T}\left(\tau_{1}, \ldots, \tau_{m}\right)=\sigma\left\{\left(\xi_{v} \leq u_{T}\left(\tau_{i}\right)\right): s \leq v \leq t, 1 \leq i \leq m\right\} .
$$

Write also

$$
\begin{aligned}
& \alpha_{T, l}\left(\tau_{1}, \ldots, \tau_{m}\right)=\sup \{\mid P(A \cap B)-P(A) P(B) \mid: \\
& A \in \mathscr{B}_{0, s}^{T}\left(\tau_{1}, \ldots, \tau_{m}\right), B \in \mathscr{B}_{s+l, T}^{T}\left(\tau_{1}, \ldots, \tau_{m}\right), \\
&\quad s \geq 0, l+s \leq T\} .
\end{aligned}
$$

Then we say that the stationary process satisfies the condition $\Delta\left(u_{T} ; \tau_{1}, \ldots\right.$, $\left.\tau_{m}\right)$ if

$$
\alpha_{T, l_{T}}\left(\tau_{1}, \ldots, \tau_{m}\right) \rightarrow 0 \quad \text { as } T \rightarrow \infty \quad \text { for some } l_{T}=o(T) .
$$

Moreover, we say that the condition $\Delta\left(u_{T}\right)$ holds for $\left\{\xi_{t}\right\}$ if $\left\{\xi_{t}\right\}$ satisfies the condition $\Delta\left(u_{T} ; \tau_{1}, \ldots, \tau_{m}\right)$ for any choice of $m$ and $\tau_{1}, \ldots, \tau_{m}>0$. It is convenient to address the characterization of the weak convergence of the excursion random measure under the condition $\Delta\left(u_{T}\right)$. However, it should be noted that the principles in our results hold in more general situations. Indeed, even stationarity can be relaxed to a certain degree. We choose not to work under the most general dependence setting to avoid complications of technical nature that are not so relevant to the main theme. This point will be apparent from the proofs and will be partially illustrated by the examples in Section 5. On the other hand, the condition $\Delta\left(u_{T}\right)$ is quite weak, and is satisfied by a large class of processes, including all strongly mixing stationary processes. Verifying whether any process satisfies any given mixing condition can of course be a nontrivial task, and we shall not spend any effort in that regard in this paper.

We first define some transformations. For $\tau \in \Re, \sigma \in \Re_{+}$, let $g_{\tau}$ and $h_{\sigma}$ be defined by

$$
\begin{aligned}
& g_{\tau}:(x, y) \rightarrow(x+\tau, y), \\
& h_{\sigma}:(x, y) \rightarrow(x / \sigma, \sigma y), \quad \Re_{+}^{2} \rightarrow \Re_{+}^{2} .
\end{aligned}
$$

Also, let $G_{\tau}$ and $H_{\sigma, \beta}, \beta \in \Re$, be defined by

$$
\begin{aligned}
G_{\tau}: \mu & \rightarrow \mu \circ g_{-\tau}, \\
H_{\sigma, \beta}: \mu & \rightarrow\left(\sigma^{\beta} \mu\right) \circ h_{1 / \sigma}, \quad \mathscr{M}\left(\Re_{+}\right) \rightarrow \mathscr{M}\left(\Re_{+}^{2}\right),
\end{aligned}
$$


where $\sigma^{\beta} \mu$ is $\sigma^{\beta}$ times $\mu$. Here, as elsewhere, the notation for a transformation and the corresponding set transformation will be the same; for example, for $B \subset \Re_{+}^{2}$,

$$
g_{\tau}(B)=\left\{g_{\tau}(x, y):(x, y) \in B\right\} .
$$

The proof of the following result is contained in Section 6 .

Proposition 2.1. Assume that $u_{T}$ satisfies conditions (U1) and (U2), and $\left\{\xi_{t}\right\}$ satisfies the condition $\Delta\left(u_{T}\right)$. Suppose there there exist positive monotone constants $\left\{a_{T}\right\}$ such that $a_{T} \zeta_{T}$ converges in distribution to some random measure $\eta \neq 0$, and

$$
\limsup _{T \rightarrow \infty} P\left\{a_{T} \zeta_{T}([0,1) \times(0,1)) \leq B\right\}>0 \text { for some } B>0 .
$$

Then $a_{T}$ is regularly varying with some index $\beta \in \Re$, and $\eta$ satisfies the conditions (A1)-(A4) below.

(A1) $G_{\tau} \circ \eta \stackrel{d}{=} \eta$ for all $\tau \in \Re$;

(A2) $H_{\sigma, \beta} \circ \eta \stackrel{d}{=} \eta$ for all $\sigma \in \mathfrak{R}_{+}$;

(A3) $P\{\eta([0,1) \times(0,1))<\infty\}>0$.

(A4) For any choice of bounded disjoint intervals $\left[a_{i}, b_{i}\right) \subset \Re, 1 \leq i \leq k$, the random measures

$$
\eta\left(\cdot \cap\left(\left[a_{i}, b_{i}\right) \times \Re_{+}\right)\right), \quad 1 \leq i \leq k,
$$

are mutually independent.

REMARK 1. As explained earlier, in most cases of interest where both (2.2) and (2.3) hold, $a_{T}$ is bounded away from zero, and then the regular variation index $\beta$ must be nonnegative. However, as illustrated by Examples 5.1 and 5.4 , generally $\beta$ can be negative as well as positive.

REMARK 2. Here (A1) and (A4), respectively, state that $\eta$ has stationary and independent increments along the direction of the horizontal axis. Condition (A2) is a type of self-similar property. It can be seen (cf. Lemma 6.4) that under (A1), (A2) and (A4), condition (A3) is equivalent to $P\{\eta([0,1) \times(0, \tau))<$ $\infty\}=1$ for all $\tau>0$.

REMARK 3. In Proposition 2.1 if $u_{T}$ satisfies (2.2), then (2.6) holds with $B=0$. In that case it is easy to see that the following condition also holds:

$$
P\{\eta([0,1) \times(0,1))=0\}>0 .
$$

We will give a characterization in Section 3 to processes satisfying (A1), (A2), (A3) or (A3'), and (A4). 
3. Characterization. Throughout this section, let $\beta \in \Re$ be a fixed constant, $\eta$ be a random measure on $\Re_{+}^{2}=(-\infty, \infty) \times(0, \infty)$. The goal of this section is to obtain a characterization for random measures $\eta$ on $\Re_{+}^{2}$ satisfying the conditions (A1)-(A4) defined in Proposition 2.1, thereby to characterize the possible limiting distributions of the excursion random measures. The proofs of Theorems 3.1 and 3.2 and Proposition 3.3 are deferred to Section 6.

We first introduce some notation. For $\sigma \in \Re_{+}$and $\phi \in \mathscr{M}\left(\Re_{+}\right)$, define

$$
T_{\sigma, \beta}(\phi)=\sigma^{\beta} \phi\left(\sigma^{-1} \cdot\right) .
$$

Let $Y$ be a mapping which maps $((x, y), \psi) \in \mathfrak{R}_{+}^{2} \times \mathscr{M}\left(\Re_{+}\right)$to $\mu \in \mathscr{M}\left(\Re_{+}^{2}\right)$ where

$$
\mu(E)=T_{y, \beta}(\psi)\left(E_{x}\right)=y^{\beta} \psi\{t / y:(x, t) \in E\}, \quad E \in \sigma\left(\Re_{+}^{2}\right) .
$$

Also define

$$
m_{\beta}(d x d y)=y^{\beta} m(d x d y), \quad(x, y) \in \Re_{+}^{2},
$$

where $m$ is Lebesgue measure on $\Re_{+}^{2}$.

THEOREM 3.1. In order for $\eta$ to satisfy (A1)-(A4), it is necessary and sufficient that $\eta$ is infinitely divisible with canonical measure $\lambda$ and deterministic part $\eta_{d}=\mathrm{cm}_{\beta}$ for some $c \in[0, \infty)$, where $c=0$ if $\beta \leq-1$, and there exists a set $\mathscr{A} \subset\left\{\psi \in \mathscr{M}\left(\Re_{+}\right) \backslash\{0\}: \psi(0,1)<\infty\right\}$, such that $\mathscr{S}:=\Upsilon\left(\Re_{+}^{2} \times \mathscr{A}\right)$ is measurable in $\mathscr{M}\left(\Re_{+}^{2}\right)$ and is a subset of the support of $\lambda$ with $\lambda\left(\mathscr{\rho}^{c}\right)=0, Y$ is oneto-one and bi measurable from $\Re_{+}^{2} \times \mathscr{A}$ to $\mathscr{A}$, and there exists a probability measure $\mathbb{P}$ on $\mathscr{A}$ such that

$$
\lambda \circ \Upsilon=m \times \mathbb{P} \quad \text { on } \Re_{+}^{2} \times \mathscr{A}
$$

and

$$
\int_{\mathscr{A}} \int_{0}^{\infty}\left(1-\exp \left(-\nu(0, \sigma) / \sigma^{\beta}\right)\right) \frac{d \sigma}{\sigma^{2}} \mathbb{P}(d \nu)<\infty
$$

REMARK 1. In the proof of Theorem 3.1 in Section 6, we will illustrate how to construct $(\mathscr{A}, \mathbb{P})$. In particular, it will be seen that the characterization in Theorem 3.1 is nonunique in that different pairs of $(\mathscr{A}, \mathbb{P})$ could appear in the decomposition (3.3). The general construction is somewhat artificial, which makes it pointless to explain it here. However, we mention that there is a rather natural construction of $(\mathscr{A}, \mathbb{P})$ in the important case where $\left(A 3^{\prime}\right)$ also holds (cf. Remark 3 of Section 2), as the following shows.

THEOREM 3.2. If (A3) is replaced by $\left(\mathrm{A}^{\prime}\right)$, then the statements of Theorem 3.1 remain valid by taking $c=0$ and

$$
\mathscr{A} \subset\left\{\psi \in \mathscr{M}\left(\Re_{+}\right) \backslash\{0\}: \psi(0, \rho)=0 \text { and } \psi[\rho, t)>0 \text { for all } t>\rho\right\},
$$

where $\rho=-\log P\{\eta([0,1) \times(0,1))=0\} \in(0, \infty)$. 
REMARK 2. We note that, by (1.1) and (3.3), the Laplace transform of a random measure $\eta$ satisfying (A1)-(A4) can be written as

$$
\begin{array}{r}
\exp \left\{-c m_{\beta} f-\int_{\mathscr{A}} \int_{\Re_{+}^{2}}\left(1-\exp \left(-\int_{0}^{\infty} f(x, y z) y^{\beta} \nu(d z)\right)\right) m(d x d y) \mathbb{P}(d \nu)\right\}, \\
f \in \mathscr{T}\left(\Re_{+}^{2}\right) .
\end{array}
$$

One useful consequence is the following alternative presentation of Theorem 3.1.

Proposition 3.3. The random measure $\eta$ described in Theorem 3.1 can be represented as

$$
\eta=c m_{\beta}+\sum Y\left(\left(X_{i}, Y_{i}\right), \gamma_{i}\right),
$$

where $c \in[0, \infty), c=0$ if $\beta \leq-1$, the $\left(X_{i}, Y_{i}\right)$ are the points of a stationary Poisson process $\Gamma$ on $\Re_{+}^{2}$ with unit intensity, the $\gamma_{i}$ are random elements in $\mathscr{A}$ distributed according to $\mathbb{P}$, and $\Gamma, \gamma_{1}, \gamma_{2}, \ldots$ are mutually independent.

In view of Proposition 3.3, a realization of the process $\eta_{r}$ (cf. Theorem 1.1) consists of an ensemble of measures confined to vertical lines. The Poisson points can be generally interpreted as "reference" points of the lines. This is especially meaningful in the important case where $\left(A 3^{\prime}\right)$ also holds, since the mass of the measure on the line at $X_{i}$ lies entirely above $\rho Y_{i}$ with $\rho$ given by Theorem 3.2.

4. The limits of the excursion random measures. We now return to the consideration of the excursion random measure described in Section 2 and will continue to use the notation defined there. By Proposition 2.1 and Theorems 3.1 and 3.2, the distributional limits of the excursion random measures are readily characterized. In this section we focus on the interpretation of the measure $\mathbb{P}$ of the limiting excursion random measure in the decomposition (3.3). We shall limit ourselves to the case where (2.2) holds. First we need to define an extended version of the condition $\Delta\left(u_{T}\right)$ : say that the condition $\Delta\left(u_{T}\right)$ holds uniformly for the stationary process $\left\{\xi_{t}\right\}$ in the sense that there are two sequences of positive integers $\left\{k_{T}\right\}$ and $\left\{l_{T}\right\}$, where $k_{T} \rightarrow \infty$, for which

$$
\lim _{T \rightarrow \infty} k_{T}\left(\alpha_{T, l_{T}}\left(\tau_{1}, \ldots, \tau_{m}\right)+l_{T} / T\right)=0
$$

holds for all $\tau_{1}, \ldots, \tau_{m}$ and all $m$.

THEOREM 4.1. Let $u_{T}$ satisfy $P\left\{M(T) \leq u_{T}(\tau)\right\} \rightarrow e^{-\tau}, \tau>0$, and let $a_{T}>$ 0 be regularly varying with index $\beta$. Let the condition $\Delta\left(u_{T}\right)$ hold uniformly for the stati onary process $\left\{\xi_{t}\right\}$. Define the random measure on $\Re_{+}$:

$$
\chi_{T}(\cdot)=a_{T}\left(u_{T}^{-1}\left(M\left(r_{T}\right)\right)\right)^{-\beta} \int_{0}^{r_{T}} I\left(\frac{u_{T}^{-1}\left(\xi_{t}\right)}{u_{T}^{-1}\left(M\left(r_{T}\right)\right)} \in \cdot\right) d t,
$$


where $r_{T}=T / k_{T}$. If on the space

$$
\tilde{\mathscr{M}}:=\left\{\psi \in \mathscr{M}\left(\Re_{+}\right): \psi(0,1)=0 \text { and } \psi[1, t)>0 \text { for all } t>1\right\},
$$

there exists some probability measure $\mathbb{P}$ to which $P\left\{\chi_{T} \in \cdot \mid M\left(r_{T}\right)>u_{T}(\tau)\right\}$ converges weakly for all $\tau>0$, then the excursion random measure $\eta_{T}=a_{T} \zeta_{T}$ converges in distribution to some nondegenerate random measure $\eta$ which has the representation given by Theorem 3.2 with $\rho=1$.

The proof of Theorem 4.1 is contained in Section 6.4. Note that by a straightforward blocking argument, the condition $\Delta\left(u_{T}\right)$ together with $P\{M(T) \leq$ $\left.u_{T}(\tau)\right\} \rightarrow e^{-\tau}, \tau>0$, implies (2.2). Some additional remarks concerning Theorem 4.1 are given below.

REMARK 1. In Theorem 4.1, the gl obal normalization $u_{T}$ controls the rate of high-level excursions while $r_{T}$ is chosen in such a way to ensure that each such excursion can be captured in a time interval nolonger than $r_{T}$. Within an interval containing a high-level excursion, the random measure $\chi_{T}$ describes the local behavior of the process relative to the supremum of the process on the interval. The result shows that, with proper local normalization, the conditional distribution of $\chi_{T}$ converges weakly to the measure $\mathbb{P}$ in the decomposition of $\eta$ in Theorem 3.2. Theorem 4.1 not only provides a tractable method of obtaining the limiting excursion random measure but also a theoretical framework for statistical modeling of local dependence.

REMARK 2. Under the assumptions of the theorem, one can also show that the weak convergence of $\eta$ implies that of $\chi_{T}$ so that the two convergence statements are equivalent. See Hsing (1993), Theorem 2.2.

REMARK 3. It is worth pointing out that the result is related to the socalled "local and global sojourn limit theorems" in Berman (1982, 1983). Consider now the Laplace-Stieltjes transform of $a_{T} \eta((0,1] \times(0, \tau))$, the normalized total excursion time of $\left\{\xi_{t}\right\}$ in $\left(u_{T}(\tau), \infty\right)$ for $t \in(0, T]$. By Remark 2 following Theorem 3.2, for $s>0$,

$$
\begin{aligned}
\lim _{T \rightarrow \infty} & E \exp \left(-s a_{T} \eta((0,1] \times(0, \tau))\right) \\
& =\exp \left\{-\int_{\mathscr{A}} \int_{0}^{\infty}\left(1-\exp \left(-s \nu(0, \tau / y) y^{\beta}\right)\right) d y \mathbb{P}(d \nu)\right\} \\
& =\exp \left\{-\int_{0}^{\infty}(1-\exp (-s x))\left(m_{+} \times \mathbb{P}\right) \circ V^{-1}(d x)\right\},
\end{aligned}
$$

where $m_{+}$is the Lebesgue measure on $\Re_{+}$and $V$ is the mapping defined by

$$
V:(y, \nu) \rightarrow \nu(0, \tau / y) y^{\beta}, \quad \Re_{+} \times A \rightarrow \Re_{+} .
$$

Comparing this with Theorem 4.1 of Berman (1983), we see that the function

$$
\left(m_{+} \times \mathbb{P}\right) \circ V^{-1}(x, \infty)
$$

plays the role of $H(x)$ or $-\Gamma^{\prime}(x)$ in Theorem 4.1 of Berman (1983). 
REMARK 4. To give a complete characterization of the limit of the excursion random measure, one should address the issue of whether there is a process $\left\{\xi_{t}\right\}$ for which a given probability measure $\mathbb{P}$ on $\tilde{\mathscr{M}}$ would appear in the decomposition of the limiting excursion random measure. This is quite difficult and so far we have not resolved it.

We illustrate in Section 5 how Theorem 4.1 can be applied to obtain limiting excursion random measures with three classes of examples.

5. Examples. This section contains four examples which illustrate the various aspects of the excursion random measure (details of proof being again deferred to Section 6). In Example 5.1 we construct a somewhat artificial example to show that the regular variation index of $a_{T}$ can take any value in the entire range $[0, \infty)$. We consider moving average stable processes in Example 5.2 and normal processes in Example 5.3. Finally, in Example 5.4 we illustrate that if the normalizing function $u_{T}$ does not reflect the extreme quantiles of $\xi_{t}, 0 \leq t<T$ then $\beta$ can be negative and the limiting excursion random measure can be nonrandom.

EXAMPLE 5.1 (Regular variation index of $a_{T}$ ). Let $\beta \geq 0$ be a constant and $X_{i}, i \in \mathrm{I}$ be i.i.d. unit exponential random variables, where I is the set of integers. Define a process $\bar{\xi}_{t}$ by

$$
\bar{\xi}_{t}=\sum_{i=-\infty}^{\infty} I(t \in[i, i+1))\left(X_{i}-(t-i) \exp \left(\beta X_{i}\right)\right), \quad t \in(-\infty, \infty) .
$$

While $\bar{\xi}_{t}$ is not stationary, $\xi_{t}:=\bar{\xi}_{t+\delta}$ is where $\delta$ is uniform on $[-1 / 2,1 / 2]$. Clearly the amounts of excursion time above any level in a large time interval by $\left\{\xi_{t}\right\}$ and $\left\{\bar{\xi}_{t}\right\}$ are asymptotically the same. Let

$$
u_{T}(\tau)=\log (T / \tau), \tau>0 \quad \text { and } u_{T}^{-1}(y)=T e^{-y}, \quad y \in(-\infty, \infty) .
$$

Then

$$
\begin{aligned}
\lim _{T \rightarrow \infty} P\left\{\sup _{0 \leq s<T} \bar{\xi}_{s} \leq u_{T}(\tau)\right\} & =\lim _{T \rightarrow \infty} P\left\{\sup _{0 \leq s<T} \xi_{s} \leq u_{T}(\tau)\right\} \\
& =\lim _{T \rightarrow \infty} P\left\{\max _{1 \leq j<[T]} X_{j} \leq u_{T}(\tau)\right\} \\
& =e^{-\tau}, \quad \tau>0 .
\end{aligned}
$$

Proposition 5.1. For the processes $\left\{\bar{\xi}_{t}\right\}$ and $\left\{\xi_{t}\right\}$ defined above, therandom measures

$$
T^{\beta} \int_{\Re} I_{E}\left(t / T, u_{T}^{-1}\left(\bar{\xi}_{t}\right)\right) d t \quad \text { and } \quad T^{\beta} \int_{\Re} I_{E}\left(t / T, u_{T}^{-1}\left(\xi_{t}\right)\right) d t, \quad E \in \sigma\left(\Re_{+}^{2}\right)
$$

both converge weakly to a limit $\eta$ which has the structure described by Theorem 3.2 with $\rho=1$ and $\mathscr{A}$ containing only the measure $\nu$, where

$$
\nu[1, x]=\log x, \quad x \geq 1 \text {. }
$$


EXAMPLE 5.2 (Moving average stable processes). We now consider the moving average stable process. Let $\left\{\xi_{t}\right\}$ be the moving average stable process described in Theorem 5.2 in Rootzén (1978); that is,

$$
\xi_{t}=\int a(t-x) d Z(x), \quad-\infty<t<\infty,
$$

where $a(t)$ is a deterministic filter satisfying various regularity conditions, and $Z$ has stationary independent stable increments with $Z(0)=0$ and $Z(1) \sim$ stable $(\sigma, \alpha, \gamma)$ where the scale parameter $\sigma=1$, the index of stability $\alpha \in(0,2)$ and the parameter of symmetry $\gamma \in[-1,1]$. We will basically follow the notation of Rootzén (1978) (with the exception of $\gamma$, the parameter of symmetry, which is denoted by $\beta$ there) to which the reader is referred for details. Thus for example, $A=\sup _{t \in \Re}(a(t) \vee 0), a=\sup _{t \in \Re}(-a(t) \vee 0)$ and $c_{\alpha}=\pi^{-1} \Gamma(\alpha) \sin (\alpha \pi / 2)$. By Corollary 5.3 of Rootzén (1978),

$$
\lim _{T \rightarrow \infty} P\left\{M(T) \leq x T^{1 / \alpha}\right\}=\exp \left(-c x^{-\alpha}\right), \quad x>0,
$$

where $c=c_{\alpha}\left(A^{\alpha}(1+\gamma)+a^{\alpha}(1-\gamma)\right)$. Thus let

$$
u_{T}(\tau)=\left(\frac{c T}{\tau}\right)^{1 / \alpha}, \quad \tau>0
$$

and

$$
u_{T}^{-1}(u)=\frac{c T}{u^{\alpha}} I(u>0)+\infty \cdot I(u \leq 0), \quad u \in \Re .
$$

PRoposition 5.2. For the moving average stable process described above, the excursion random measure

$$
\int_{\Re} I_{E}\left(t / T, u_{T}^{-1}\left(\xi_{t}\right)\right) d t, \quad E \in \sigma\left(\Re_{+}^{2}\right),
$$

converges in distribution to $\eta$, which has thestructuredescribed by Theorem 3.2 with $\rho=1, \beta=0$ and

$$
\mathbb{P}\left\{\nu_{+}\right\}=\frac{A^{\alpha}(1+\gamma)}{A^{\alpha}(1+\gamma)+a^{\alpha}(1-\gamma)}, \quad \mathbb{P}\left\{\nu_{-}\right\}=\frac{a^{\alpha}(1-\gamma)}{A^{\alpha}(1+\gamma)+a^{\alpha}(1-\gamma)},
$$

where $\nu_{+}[1, x]=\int_{-\infty}^{\infty} I\left(a(t) \geq A x^{-1 / \alpha}\right) d t, \nu_{-}[1, x]=\int_{-\infty}^{\infty} I\left(-a(t) \leq a x^{-1 / \alpha}\right) d t$, for $x \geq 1$.

EXAMPLE 5.3 (Normal processes). Consider in this example the stationary normal process $\left\{\xi_{t}\right\}$, standardized to have zero mean and unit variance. Assume that the covariance $r(t)=E \xi_{0} \xi_{t}$ satisfies

$$
r(t)=1-\lambda_{2} t^{2} / 2+\lambda_{4} t^{4} / 4 !+o\left(t^{4}\right) \text { as } t \rightarrow 0
$$

and $r(t)$ tends to 0 fast enough as $t \rightarrow \infty$ [cf. Ibragimov and Rozanov (1978)] so that $\left\{\xi_{t}\right\}$ is strongly mixing. The conditions on $r(t)$ at both 0 and $\infty$ could possibly be weakened. In particular, we assume strong-mixing here so that we can readily apply Theorem 4.1. We have shown elsewhere that the excursion 
random measure converges in distribution by a different method based on the condition $r(t) \log t \rightarrow 0$ as $t \rightarrow \infty$. [cf. Hsing and Leadbetter (1997)].

By Rice's formula [Theorem 7.3.2 of Leadbetter, Lindgren and Rootzén (1983)], the mean number of upcrossings of a level $u$ by $\xi_{t}$ in a unit interval is

$$
\mu(u)=\frac{\lambda_{2}^{1 / 2}}{2 \pi} \exp \left(-\frac{u^{2}}{2}\right), \quad u \in \Re .
$$

Let

$$
u_{T}(\tau)=\left(2 \log \frac{\lambda_{2}^{1 / 2} T}{2 \pi \tau}\right)^{1 / 2}, \quad \tau>0
$$

so that $T \mu\left(u_{T}(\tau)\right)=\tau, \tau>0$, and

$$
u_{T}^{-1}(u)=\frac{T \lambda_{2}^{1 / 2}}{2 \pi} \exp \left(-\frac{u^{2}}{2}\right) I(u>0)+\infty \cdot I(u \leq 0), \quad u \in \Re .
$$

By Theorem 11.1.5 of Leadbetter, Lindgren and Rootzén (1983),

$$
\lim _{T \rightarrow \infty} P\left\{M(T) \leq u_{T}(\tau)\right\}=e^{-\tau}, \quad \tau>0 .
$$

Proposition 5.3. For the stationary normal process described above, and with $a_{T}=u_{T}(1)$, the excursion random measure

$$
a_{T} \int_{\Re} I_{E}\left(t / T, u_{T}^{-1}\left(\xi_{t}\right)\right) d t, \quad E \in \sigma\left(\Re_{+}^{2}\right)
$$

converges in distribution to $\eta$ which has the structure described by Theorem 3.2 with $\rho=1, \beta=0$, and $\mathscr{A}$ containing only the measure $\nu$ satisfying

$$
\nu[1, y]=2\left(2 \lambda_{2}^{-1} \log y\right)^{1 / 2}, \quad y \geq 1 .
$$

EXAMPLE 5.4 (Degenerate limit). The following result provides a contrast and shows that if the normalization $u_{T}$ is not chosen appropriately, $a_{T}$ may tend to 0 , in which case a degenerate limiting excursion random measure is possible.

Proposition 5.4. Let $a_{T}>0$ be regularly varying with index $\beta \in(-1,0)$ and let $a_{T}$ and $u_{T}$ be such that

$$
a_{T} T P\left\{\xi_{1}>u_{T}(\tau)\right\} \rightarrow \tau^{\beta+1} \text { for all } \tau>0 .
$$

Also let the condition $\Delta\left(u_{T} ; \tau\right)$ hold for $\left\{\xi_{t}\right\}$ for all $\tau>0$, such that for each $\tau>0$ there exist $k_{T}, l_{T} \geq 1$ for which

$$
\underset{n \rightarrow \infty}{\limsup } a_{T} T / k_{T}<\infty \text { and } \lim _{T \rightarrow \infty} k_{T}\left(\alpha_{T, l_{T}}(\tau)+l_{T} / T\right)=0 .
$$

Then $a_{T} \zeta_{T} \stackrel{p}{\rightarrow}(\beta+1) m_{\beta}$, where $m_{\beta}$ is defined by (3.2).

6. Technical details. Sections 6.1-6.6 contain, respectively, the proofs of Proposition 2.1, Theorem 3.1, Theorem 3.2, Proposition 3.3, Theorem 4.1 and those for Examples 5.1 to 5.4. 
6.1. Proof of Proposition 2.1. We first state a lemma.

LEMMA 6.1. Suppose $\tau_{1}, \ldots, \tau_{m}$ are positive reals, that the condition $\Delta\left(u_{T}\right.$; $\left.\tau_{1}, \ldots, \tau_{m}\right)$ holds for $\left\{\xi_{t}\right\}$, and that $\left\{k_{T}\right\}$ is a collection of positive integers satisfying

$$
\lim _{T \rightarrow \infty} k_{T}\left(\alpha_{T, l_{T}}\left(\tau_{1}, \ldots, \tau_{m}\right)+l_{T} / T\right)=0 .
$$

Let $I_{i}\left(=I_{i}(T)\right), 1 \leq i \leq k_{T}$, be disjoint subintervals of $[0,1)$ with lengths $\geq$ $l_{T} / T, \cup_{1}^{k_{T}} I_{i}=[0,1)$ and $J_{j}=\left(0, \tau_{j}\right), 1 \leq j \leq m$. Then for any nonnegative constants $s_{i j}\left(=s_{i j}(T)\right), 1 \leq i \leq k_{T}, 1 \leq j \leq m$,

$$
\begin{aligned}
E \exp & \left(-\sum_{i=1}^{k_{T}} \sum_{j=1}^{m} s_{i j} \zeta_{T}\left(I_{i} \times J_{j}\right)\right) \\
- & \prod_{i=1}^{k_{T}} E \exp \left(-\sum_{j=1}^{m} s_{i j} \zeta_{T}\left(I_{i} \times J_{j}\right)\right) \rightarrow 0 \text { as } T \rightarrow \infty .
\end{aligned}
$$

PRoof. For any fixed choice of $\left\{s_{i j}\right\}$ define the random measure

$$
\zeta_{T}^{\prime}(B):=\sum_{i=1}^{k_{T}} \sum_{j=1}^{m} s_{i j} \zeta_{T}\left(\left(B \cap I_{i}\right) \times J_{j}\right), \quad B \subset[0,1) .
$$

Thus $\zeta_{T}^{\prime}$ is a random measure satisfying the conditions of Lemma 2.2 of Leadbetter and Hsing (1990), from which the result follows by taking $B=[0,1)$.

REMARK 1. In view of (2.4), (6.1) clearly holds for bounded $k_{T}$, and moreover $k_{T}$ can be chosen to tend to $\infty$ and satisfy (6.1).

Proof OF Proposition 2.1. That $\eta$ satisfies (A1) follows readily from the stationarity of $\left\{\xi_{t}\right\}$. Next we show that $a_{T}$ is regularly varying and that (A2) holds. For $\sigma$ and $T>0$, define a one-one transformation

$$
h_{\sigma, T}(x, y)=\left(x / \sigma, u_{\sigma T}^{-1} \circ u_{T}(y)\right), \quad(x, y) \in \mathfrak{R}_{+}^{2} .
$$

It follows from (U2) that

$$
\lim _{T \rightarrow \infty} h_{\sigma, T}(x, y)=h_{\sigma}(x, y), \quad(x, y) \in \Re_{+}^{2},
$$

where $h_{\sigma}$ is defined in (2.5). By definition, for $E \in \sigma\left(\Re_{+}^{2}\right)$,

$$
\zeta_{T}(E)=\int I_{E}\left(t / T, u_{T}^{-1}\left(\xi_{t}\right)\right) d t
$$

and

$$
\begin{aligned}
\zeta_{\sigma T}(E) & =\int I_{E}\left(\frac{t}{\sigma T}, u_{\sigma T}^{-1}\left(\xi_{t}\right)\right) d t=\int I_{E}\left(\frac{t}{\sigma T}, u_{\sigma T}^{-1} \circ u_{T} \circ u_{T}^{-1}\left(\xi_{t}\right)\right) d t \\
& =\int I_{E}\left(h_{\sigma, T}\left(\frac{t}{T}, u_{T}^{-1}\left(\xi_{t}\right)\right)\right) d t=\zeta_{T} \circ h_{\sigma, T}^{-1}(E) .
\end{aligned}
$$


Thus

$$
\zeta_{T}=\zeta_{\sigma T} \circ h_{\sigma, T}, \quad \sigma, T>0
$$

It follows that

$$
a_{T} \zeta_{T}=a_{T} \zeta_{\sigma T} \circ h_{\sigma, T}=\frac{a_{T}}{a_{\sigma T}} a_{\sigma T} \zeta_{\sigma T} \circ h_{\sigma, T}, \quad \sigma, T>0 .
$$

Since $a_{\sigma T} \zeta_{\sigma T} \rightarrow_{d} \eta$ by assumption, it follows from (6.2) and the continuous mapping theorem that

$$
a_{\sigma T} \zeta_{\sigma T} \circ h_{\sigma, T} \rightarrow_{d} \eta \circ h_{\sigma}, \sigma>0 \quad \text { as } T \rightarrow \infty .
$$

By (6.3), this implies that $a_{T} / a_{\sigma T}$ converges for each $\sigma>0$ as $T \rightarrow \infty$. There fore $a_{T}$ is regularly varying and $a_{T} / a_{\sigma T} \rightarrow \sigma^{-\beta}$ for some $\beta$ (cf. Resnick, 1987). Then (A2) also follows from (6.3).

By Lemma 3.1 of Hsing (1987), $a_{T} \zeta_{T}([0,1) \times(\varepsilon, 1) \stackrel{d}{\rightarrow} \eta([0,1) \times(\varepsilon, 1))$ for all $\varepsilon>0$. Thus, by (2.6) and Billingsley (1968), Theorem 2.1, for some $B>0$,

$$
\begin{aligned}
P\{\eta([0,1) \times(0,1)) \leq B\} & =\lim _{\varepsilon \rightarrow 0} P\{\eta([0,1) \times(\varepsilon, 1)) \leq B\} \\
& \geq \limsup _{\varepsilon \rightarrow 0} \limsup _{T \rightarrow \infty} P\left\{a_{T} \zeta_{T}([0,1) \times(\varepsilon, 1)) \leq B\right\} \\
& \geq \limsup _{T \rightarrow \infty} P\left\{a_{T} \zeta_{T}([0,1) \times(0,1)) \leq B\right\}>0 .
\end{aligned}
$$

This shows (A3). Next let $I_{i}=\left[a_{i}, b_{i}\right), 1 \leq i \leq k$, and $J_{j}=\left(0, \tau_{j}\right), 1 \leq j \leq m$, where $-\infty<a_{i}<b_{i}<\infty$ with the $\left[a_{i}, b_{i}\right)$ disjoint and $0<\tau_{j}<\infty$. To show (A4) it suffices to show for all $s_{i j} \geq 0$,

$$
E \exp \left(-\sum_{i=1}^{k} \sum_{j=1}^{m} s_{i j} \eta\left(I_{i} \times J_{j}\right)\right)=\prod_{i=1}^{k} E \exp \left(-\sum_{j=1}^{m} s_{i j} \eta\left(I_{i} \times J_{j}\right)\right) .
$$

In doing so, note that by (A1) and (A2) we need verify (6.4) only for the case where the $I_{i}$ 's are contained in $[0,1)$. By Lemma 6.1, the condition $\Delta\left(u_{T}\right)$ implies that as $T \rightarrow \infty$,

$$
\begin{aligned}
E \exp & \left(-a_{T} \sum_{i=1}^{k} \sum_{j=1}^{m} s_{i j} \zeta_{T}\left(I_{i} \times J_{j}\right)\right) \\
- & \prod_{i=1}^{k} E \exp \left(-a_{T} \sum_{j=1}^{m} s_{i j} \zeta_{T}\left(I_{i} \times J_{j}\right)\right) \rightarrow 0
\end{aligned}
$$

Since $a_{T} \zeta_{T} \rightarrow_{d} \eta$, applying Lemma 6.1.2 of Kallenberg (1983) and Lemma 3.1 of Hsing (1987) we get

$$
\left(a_{T} \zeta_{T}\left(I_{i} \times J_{j}\right), 1 \leq i \leq k, 1 \leq j \leq m\right) \rightarrow_{d}\left(\eta\left(I_{i} \times J_{j}\right), 1 \leq i \leq k, 1 \leq j \leq m\right) .
$$

Thus (6.4) follows from (6.5). This completes the proof. 
6.2. The proof of Theorem 3.1. In this subsection we give a detailed proof of Theorem 3.1. Since the proof is rather long, we divide it into several parts. First, the sufficiency part of the theorem is relatively straightforward. In fact, it will be quite obvious after the proof of necessity. Therefore we shall focus on the more difficult part of the proof and begin by assuming that $\eta$ is a random measure on $\mathfrak{R}_{+}^{2}$, satisfying conditions (A1)-(A4).

LEMMA 6.2. The term $\eta$ is infinitely divisible Write, with the notation introduced in Theorem 1.1, $\eta=\eta_{d}+\eta_{r}$. Then conditions (A1)-(A4) hold with $\eta$ replaced by $\eta_{d}$ and $\eta_{r}$.

Proof. To show infinite divisibility only requires (A1) and (A4). It suffices to show that $\left(\eta\left(E_{m}\right), 1 \leq m \leq k\right)$ is infinitely divisible as a random vector for each integer $k$ and bounded sets $E_{1}, \ldots, E_{k}$ of the form $[a, b) \times[c, d) \subset \Re_{+}^{2}$ [cf. Kallenberg (1983), Lemma 6.3]. Moreover by (A4), it suffices to show that $\left(\eta\left(E_{i j}\right), 1 \leq i \leq I, 1 \leq j \leq J\right)$ is infinitely divisible as a random vector for any integers $I$ and $J$, and bounded sets $E_{i j}=\left[a_{i}, b_{i}\right) \times\left[c_{j}, d_{j}\right) \subset \Re_{+}^{2}$, where the $\left[a_{i}, b_{i}\right)$ 's are disjoint. For each $n \geq 1$, write

$$
E_{i j k}^{(n)}=\left[a_{i}+\frac{(k-1)\left(b_{i}-a_{i}\right)}{n}, a_{i}+\frac{k\left(b_{i}-a_{i}\right)}{n}\right) \times\left[c_{j}, d_{j}\right) .
$$

By (A1) and (A4), the $n$ random vectors $\left(\eta\left(E_{i j k}^{(n)}\right), 1 \leq i \leq I, 1 \leq j \leq J\right)$, $1 \leq k \leq n$, are i.i.d., showing that $\eta$ is infinitely divisible. Clearly, (A3) and (A4) must both hold for $\eta$ replaced by $\eta_{d}$ and $\eta_{r}$. To show that the same can be said for (A1) and (A2), simply notice that

$$
\begin{gathered}
G_{\tau}\left(\eta_{d}\right)=\left(G_{\tau}(\eta)\right)_{d} \stackrel{d}{=} \eta_{d}, \\
H_{\sigma, \beta}\left(\eta_{d}\right)=\left(H_{\sigma, \beta}(\eta)\right)_{d} \stackrel{d}{=} \eta_{d} .
\end{gathered}
$$

This completes the proof.

In view of Lemma 6.2 and Theorem 1.1, to characterize $\eta$, we can focus on the structure of $\eta_{d}$ and $\eta_{r}$ (equivalently, $\alpha$ and $\lambda$ ). In the following let $m_{ \pm}, m_{+}$ and $m$, respectively, be Lebesgue measures on $\Re_{,} \Re_{+}$and $\Re_{+}^{2}$. Recall that $m_{\beta}$ is the measure on $\Re_{+}^{2}$ defined by (3.2). The deterministic part $\eta_{d}$ is completely characterized by the following.

Lemma 6.3. $\eta_{d}=c m_{\beta}$ for some $c \in[0, \infty)$, where $c=0$ if $\beta \leq-1$.

Proof. Define

$$
\tilde{\eta}_{d}(E)=\int_{E} y^{-\beta} \eta_{d}(d x, d y), \quad E \in \sigma\left(\Re_{+}^{2}\right) .
$$


By (A2),

$$
\begin{aligned}
\left(H_{\sigma, 0} \circ \tilde{\eta}_{d}\right)(E) & =\tilde{\eta}_{d} \circ h_{1 / \sigma}(E)=\int_{E}(y / \sigma)^{-\beta}\left(\eta_{d} \circ h_{1 / \sigma}\right)(d x, d y) \\
& =\int_{E} y^{-\beta}\left(H_{\sigma, \beta} \circ \eta_{d}\right)(d x, d y)=\int_{E} y^{-\beta} \eta_{d}(d x, d y)=\tilde{\eta}_{d}(E) .
\end{aligned}
$$

Thus it is easily seen that

$$
\tilde{\eta}_{d}=G_{\tau} \circ \tilde{\eta}_{d}=H_{\sigma, 0} \circ \tilde{\eta}_{d} \quad \text { for all }(\tau, \sigma) \in \Re_{+}^{2} .
$$

It follows from Lemma 2.5 of Hsing (1987) that $\tilde{\eta}_{d}$ is a constant multiple of Lebesgue measure $m$, and hence $\eta_{d}(d x, d y)=c m_{\beta}(d x, d y)$ for some $c \geq 0$. However, if $\beta \leq-1$ then $m_{\beta}([0,1) \times(0,1))=\infty$ so that $c=0$ by (A3).

The rest of the proof focuses on the consideration of $\eta_{r}$, or equivalently, the canonical measure $\lambda$.

LEMma 6.4. The following hold.

(i) $P\left\{\eta_{r}([0,1) \times(0,1))<\infty\right\}=1$; equivalently, $\lambda\left\{\mu \in \mathscr{M}\left(\Re_{+}^{2}\right) \backslash\{0\}\right.$ : $\mu([0,1) \times(0,1))=\infty\}=0$.

(ii) $P\left\{\eta_{r}([0,1) \times(0, \varepsilon))=0\right\}$ is either identically equal to 0 or strictly positive and tends to 1 as $\varepsilon$ tends to 0 ; equivalently, $\lambda\left\{\mu \in \mathscr{M}\left(\Re_{+}^{2}\right) \backslash\{0\}: \mu([0,1) \times\right.$ $(0, \varepsilon))>0\}$ is either identically equal to $\infty$ or strictly positive and tends to 0 as $\varepsilon$ tends to 0; equivalently, $P\left\{\eta_{r}([0,1) \times(0,1))=0\right\}=0$ or $>0$.

Proof. By (A1), (A2) and (A4) (cf. Lemma 6.2),

$$
\begin{aligned}
P^{2}\left\{\eta_{r}([0,1) \times(0,1))<\infty\right\} & =P\left\{\eta_{r}([0,2) \times(0,1))<\infty\right\} \\
& =P\left\{2^{-\beta} \eta_{r}([0,1) \times(0,2))<\infty\right\} \\
& =P\left\{\eta_{r}([0,1) \times(0,1))<\infty\right\},
\end{aligned}
$$

where the last equality follows from the fact that $[0,1) \times[1,2)$ is bounded and hence $P\left\{\eta_{r}([0,1) \times[1,2))<\infty\right\}=1$. This implies that $P\left\{\eta_{r}([0,1) \times(0,1))<\right.$ $\infty\}=1$ by (A3). Using the Laplace transform representation (2.1), it follows by dominated convergence that

$$
\begin{aligned}
\lambda\{\mu & \left.\in \mathscr{M}\left(\Re_{+}^{2}\right) \backslash\{0\}: \mu([0,1) \times(0,1))=\infty\right\} \\
& =-\lim _{s \rightarrow 0} \int(1-\exp (-s \mu([0,1) \times(0,1)))) \lambda(d \mu) \\
& =-\lim _{s \rightarrow 0} \log \mathscr{E} \exp \left(-s \eta_{r}([0,1) \times(0,1))\right. \\
& =-\log P\left\{\eta_{r}([0,1) \times(0,1))<\infty\right\}=0 .
\end{aligned}
$$

This proves (i). To prove (ii), note that by (A1), (A2) and (A4),

$$
\begin{aligned}
P\left\{\eta_{r}\left([0,1) \times\left(0, \frac{1}{n}\right)\right)=0\right\} & =P\left\{n^{-\beta} \eta_{r}\left(\left[0, \frac{1}{n}\right) \times(0,1)\right)=0\right\} \\
& =P^{1 / n}\left\{\eta_{r}([0,1) \times(0,1))=0\right\},
\end{aligned}
$$


which is identically equal to zero or tends to one according as $P\left\{\eta_{r}([0,1) \times\right.$ $(0,1))=0\}=0$ or $>0$. Then (ii) follows by monotone convergence from

$$
\begin{aligned}
\lambda\{\mu & \left.\in \mathscr{M}\left(\Re_{+}^{2}\right) \backslash\{0\}: \mu([0,1) \times(0, \varepsilon))>0\right\} \\
& =\lim _{s \rightarrow \infty} \int(1-\exp (-s \mu([0,1) \times(0, \varepsilon)))) \lambda(d \mu) \\
& =-\lim _{s \rightarrow \infty} \log \mathscr{E} \exp \left(-s \eta_{r}([0,1) \times(0, \varepsilon))\right) \\
& =-\log P\left\{\eta_{r}([0,1) \times(0, \varepsilon))=0\right\} .
\end{aligned}
$$

(i) of Lemma 6.4 shows that under (A1), (A2) and (A4), the condition (A3) is equivalent to $P\{\eta([0,1) \times(0,1))<\infty\}=1$. (ii) of Lemma 6.4 describes the dichotomy of $\eta$ satisfying $\left(\mathrm{A}^{\prime}\right)$ and the complement of $\left(\mathrm{A}^{\prime}\right)$ in (A3). We will see below that the characterization problem is more straightforward under (A3').

Let $\Theta \operatorname{map}(x, \psi)$ in $\Re \times \mathscr{M}\left(\Re_{+}\right)$to $\mu$ in $\mathscr{M}\left(\Re_{+}^{2}\right)$ satisfying $\mu(E)=\psi\left(E_{x}\right)=$ $\psi\{t:(x, t) \in E\}, E \subset \mathfrak{R}_{+}^{2}$. Also, for convenience, say that the condition $V(\beta)$ holds for some set $\mathscr{C} \subset \mathscr{M}\left(\Re_{+}\right) \backslash\{0\}$ if

$$
\int_{0}^{1}\left(1-\exp \left(-\psi(0, \sigma) / \sigma^{\beta}\right)\right) \sigma^{-1} d \sigma<\infty, \quad \psi \in \mathscr{C}
$$

and $\mathscr{C}$ is invariant under the family of transformations $\left\{T_{\sigma, \beta}, \sigma>0\right\}$ [defined by (3.1)] in the sense that $\psi \in \mathscr{C} \Rightarrow T_{\sigma, \beta}(\psi) \in \mathscr{C}$ for all $\sigma>0$ [equivalently, $T_{\sigma, \beta}(\mathscr{b})=\mathscr{b}$ for all $\sigma>0$ ].

LEMMA 6.5. There exists $\mathscr{B} \subset\left\{\psi \in \mathscr{M}\left(\Re_{+}\right) \backslash\{0\}: \psi(0,1)<\infty\right\}$ measurable in $\mathscr{M}\left(\Re_{+}\right)$and satisfying the condition $V(\beta)$, such that $\mathscr{P}:=\Theta(\Re \times \mathscr{B})$ is measurable in $\mathscr{M}\left(\Re_{+}^{2}\right)$ and is contained in the support of $\lambda$ with $\lambda\left(\mathscr{\rho}^{c}\right)=0, \Theta$ is one-one and bimeasurable as a mapping from $\Re \times \mathscr{B}$ to $\mathscr{P}$ and

$$
\lambda \circ \Theta=m_{ \pm} \times \mathrm{Q} \quad \text { on } \Re \times \mathscr{B},
$$

where $m_{ \pm}$is Lebesgue measure on $\Re$ and $Q$ is a measure on $\mathscr{B}$ such that

$$
\int_{\mathscr{B}}(1-\exp (-\psi(0,1))) \mathrm{Q}(d \psi)<\infty
$$

with $T_{\sigma, \beta}$ defined by (3.1).

Proof. Define

$$
\Lambda=\Theta\left(\Re \times\left\{\psi \in \mathscr{M}\left(\Re_{+}\right) \backslash\{0\}: \psi(0,1)<\infty\right\}\right) .
$$

We first show that $\lambda\left(\Lambda^{c}\right)=0$. Then $\Lambda$ can be written as $A \cap B$ where

$$
\begin{aligned}
& A=\left\{\mu \in \mathscr{M}\left(\Re_{+}^{2}\right) \backslash\{0\}: \mu\left(\{x\} \times \Re_{+}\right)^{c}=0 \text { for exactly one } x \in \Re\right\}, \\
& B=\left\{\mu \in \mathscr{M}\left(\Re_{+}^{2}\right) \backslash\{0\}: \mu(\Re \times(0,1))<\infty\right\} .
\end{aligned}
$$

Since $\Lambda^{c}=A^{c} \cup\left(A \cap B^{c}\right)$, it is sufficient to show

$$
\lambda\left(A^{c}\right)=\lambda\left(A \cap B^{c}\right)=0 .
$$


Let $A_{m n}=\left\{\mu \in \mathscr{M}\left(\Re_{+}^{2}\right) \backslash\{0\}: \mu\left(\left[k / 2^{n},(k+1) / 2^{n}\right) \times(0, m)\right)=0\right.$ for all but possibly one $k$ in I $\}$, where I is the set of all integers. Then $A_{m n}$ is nonincreasing in $m$ for each $n, \bigcap_{m=1}^{\infty} A_{m n}$ is nonincreasing in $n$ and $A=\bigcap_{n=1}^{\infty} \bigcap_{m=1}^{\infty} A_{m n}$. Thus

$$
\begin{aligned}
\lambda\left(A^{c}\right) & =\lim _{n \rightarrow \infty} \lim _{m \rightarrow \infty} \lambda\left(A_{m n}^{c}\right) \\
& \leq \lim _{n \rightarrow \infty} \lim _{m \rightarrow \infty} \sum_{i \neq j} \lambda\left\{\mu \in \mathscr{M}\left(\Re_{+}^{2}\right) \backslash\{0\}: \mu\left(\left[\frac{i}{2^{n}}, \frac{i+1}{2^{n}}\right) \times(0, m)\right)>0\right. \\
& \text { and } \left.\mu\left(\left[\frac{j}{2^{n}}, \frac{j+1}{2^{n}}\right) \times(0, m)\right)>0\right\} .
\end{aligned}
$$

By (A4) and Lemma 6.4(i), we can apply 2.2.12 of Matthes, Kerstan and Mecke (1978) [cf. Lemma 7.3 of Kallenberg (1983)] to conclude that

$$
\begin{aligned}
& \lambda\left\{\mu \in \mathscr{M}\left(\Re_{+}^{2}\right) \backslash\{0\}: \mu(\right. {\left.\left[\frac{i}{2^{n}}, \frac{i+1}{2^{n}}\right) \times(0, m)\right)>0 \text { and } } \\
&\left.\mu\left(\left[\frac{j}{2^{n}}, \frac{j+1}{2^{n}}\right) \times(0, m)\right)>0\right\}=0
\end{aligned}
$$

for each $i, n$ and $m$. Thus $\lambda\left(A^{c}\right)=0$. Similarly

$$
A \cap B^{c} \subset \bigcup_{m=-\infty}^{\infty}\left\{\mu \in \mathscr{M}\left(\Re_{+}^{2}\right) \backslash\{0\}: \mu([m-1, m) \times(0,1))=\infty\right\} .
$$

It follows readily from Lemma 6.2 that

$$
\lambda \circ G_{\tau}=\lambda \text { for all } \tau \in \Re .
$$

Thus Lemma 6.4(i) implies that

$$
\lambda\left(A \cap B^{c}\right) \leq \sum_{m=-\infty}^{\infty} \lambda\left\{\mu \in \mathscr{M}\left(\Re_{+}^{2}\right) \backslash\{0\}: \mu([m-1, m) \times(0,1))=\infty\right\}=0 .
$$

This shows $\lambda\left(\Lambda^{c}\right)=0$.

Now, by (6.9), if $\Theta(\tau, \psi)$ is in the support of $\lambda$ for some $\tau \in \Re$, then $\Theta(\tau, \psi)$ is in the support of $\lambda$ for all $\tau \in \Re$. Therefore, support $(\lambda)=\Theta(\Re \times \mathscr{C})$ for some closed set $\mathscr{b} \subset \mathscr{M}\left(\Re_{+}\right) \backslash\{0\}$. Write

$$
\tilde{\mathcal{S}}=\operatorname{support}(\lambda) \cap \Lambda \text {. }
$$

In view of the definition of $\Lambda$, we conclude that

$$
\tilde{\mathscr{\rho}}=\Theta(\Re \times \tilde{\mathscr{B}}),
$$

where $\tilde{\mathscr{B}}=\{\psi \in \mathscr{C}: \psi(0,1)<\infty\}$. Clearly $\tilde{\mathscr{S}}$ and $\tilde{\mathscr{B}}$ are, respectively, measurable in $\mathscr{M}\left(\Re_{+}^{2}\right)$ and $\mathscr{M}\left(\Re_{+}\right), \tilde{\mathscr{\rho}}$ is contained in the support of $\lambda$ with $\lambda\left(\tilde{\mathscr{\rho}}^{c}\right)=0$ and $\Theta$ is one-one and bimeasurable from $\Re \times \tilde{\mathscr{B}}$ to $\tilde{\mathscr{P}}$.

Next fix $E \in \sigma(\tilde{\mathscr{B}})$ and define the set function

$$
\rho_{E}(B)=\lambda \circ \Theta(B \times E), \quad B \in \sigma(\Re) .
$$


Let $B_{i}, i \geq 1$ be mutually disjoint sets in $\sigma(\Re)$. Since $\lambda$ is a measure and $\Theta$ is one-one and bimeasurable, we have

$$
\rho_{E}\left(\bigcup_{i=1}^{\infty} B_{i}\right)=\lambda\left(\bigcup_{i=1}^{\infty} \Theta\left(B_{i} \times E\right)\right)=\sum_{i=1}^{\infty} \rho_{E}\left(B_{i}\right) .
$$

Thus $\rho_{E}(\cdot)$ is a measure on $\Re$. By (6.9), for each $B \in \sigma(\Re)$,

$$
\rho_{E}(B+\tau)=\rho_{E}(B), \quad \tau \in \Re
$$

This shows that $\rho_{E}$ can be written as $\tilde{Q}(E) \times m_{ \pm}$where $\tilde{Q}(E)$ is a nonnegative constant. Next regarding Q as a set function on $\sigma(\tilde{\mathscr{B}})$, it can be shown as before that $\tilde{Q}$ is a measure, and hence

$$
\lambda \circ \Theta=m_{ \pm} \times \tilde{Q} \quad \text { on } \Re \times \tilde{\mathscr{B}} .
$$

Applying (i) of Lemma 6.4, (6.10), and the canonical representation in Theorem 1.1, it follows that

$$
\int_{\tilde{\mathscr{B}}}(1-\exp (-\psi(0,1))) \tilde{\mathrm{Q}}(d \psi)<\infty .
$$

Now, applying (A2) and (6.10) twice, it follows that

$$
\begin{aligned}
\sigma\left(m_{ \pm} \times \tilde{\mathrm{Q}}\right)(B \times E) & =\left(m_{ \pm} \times \tilde{\mathrm{Q}}\right)(\sigma B \times E)=\lambda \circ \Theta(\sigma B \times E) \\
= & \lambda \circ H_{\sigma, \beta} \circ \Theta(\sigma B \times E) \\
= & \lambda \circ \Theta\left(B \times T_{\sigma, \beta}(E)\right)=\left(m_{ \pm} \times \tilde{\mathrm{Q}}\right)\left(B \times T_{\sigma, \beta}(E)\right), \\
& B \in \sigma(\Re), E \in \sigma(\tilde{\mathscr{B}}),
\end{aligned}
$$

showing that

$$
\tilde{\mathrm{Q}}=\sigma^{-1} \tilde{\mathrm{Q}} \circ T_{\sigma, \beta}, \quad \sigma>0 .
$$

It is then straightforward to conclude that $\tilde{\mathscr{B}}$ (which is the support of $\tilde{Q}$ ) is invariant under the family of transformations $\left\{T_{\sigma, \beta}, \sigma>0\right\}$. (See the explanation following (6.6).) Moreover, (6.11) and (6.12) imply that

$$
\begin{aligned}
\infty & >\int_{\tilde{\mathscr{B}}}(1-\exp (-\psi(0,1))) \tilde{\mathrm{Q}}(d \psi) \\
& =\int_{0}^{1} \int_{\tilde{\mathscr{B}}}(1-\exp (-\psi(0,1))) \tilde{\mathrm{Q}}\left(d T_{\sigma, \beta}(\psi)\right) \frac{d \sigma}{\sigma} \\
& =\int_{\tilde{\mathscr{B}}} \int_{0}^{1}\left(1-\exp \left(-\psi(0, \sigma) / \sigma^{\beta}\right)\right) \frac{d \sigma}{\sigma} \tilde{\mathrm{Q}}(d \psi) .
\end{aligned}
$$

Thus (6.6) holds for all $\psi \in \tilde{\mathscr{B}}$ except a set $\mathscr{D}$ of Q-measure 0, where

$$
\mathscr{D}=\left\{\psi \in \tilde{\mathscr{B}}: \int_{0}^{1}\left(1-\exp \left(-\psi(0, \sigma) / \sigma^{\beta}\right)\right) \frac{d \sigma}{\sigma}=\infty\right\} .
$$

Note that $\mathscr{D}$ (and hence $\left.\mathscr{D}^{c}\right)$ is also measurable in $\mathscr{M}\left(\Re_{+}\right)$and invariant under $\left\{T_{\sigma, \beta}, \sigma>0\right\}$. 
Finally, making use of the above, $\mathscr{B}:=\tilde{\mathscr{B}} \cap \mathscr{D}^{c}, \mathscr{P}:=\Theta(\Re \times \mathscr{B})$ and $\mathrm{Q}:=$ the restriction of $\tilde{Q}$ to $\mathscr{B}$ satisfy the requirements of the lemma.

We now continue the proof with the notation developed in Lemma 6.4. For convenience, call a function $s$ from $\mathscr{B}$ to $\mathrm{R}_{+}$a scale function for $\mathscr{B}$ if it is measurable and

$$
s\left(T_{\sigma, \beta}(\psi)\right)=\sigma s(\psi), \quad \sigma>0, \psi \in \mathscr{B} .
$$

There exist infinitely many different scale functions for $\mathscr{B}$. A "good" scale function makes the decomposition of (3.3) more informative. The proof of Theorem 3.1 does not depend on the particular choice of this function. However, we now show that there exists at least one leading to the set of criteria as in Theorem 3.1. Define

$$
g_{\psi}(y)=\int_{0}^{y}\left(1-\exp \left(-\psi(0, \sigma) / \sigma^{\beta}\right)\right) \sigma^{-1} d \sigma, \quad y>0 .
$$

By a change of variables, it is readily seen that

$$
g_{T_{\sigma, \beta}(\psi)}(y)=g_{\psi}(y / \sigma), \quad y>0, \sigma>0
$$

and it is clear that $g_{\psi}(y)<\infty$ for all $y>0$ and $\psi \in \mathscr{B}$. Consider the function

$$
s: \psi \rightarrow \inf \left\{y>0: g_{\psi}(y) \geq 1 \wedge g_{\psi}(\infty) / 2\right\}, \quad \mathscr{B} \rightarrow \mathfrak{R}_{+} .
$$

By (6.14) for any $\sigma>0$,

$$
\begin{aligned}
s\left(T_{\sigma, \beta}(\psi)\right) & =\inf \left\{y>0: g_{T_{\sigma, \beta}(\psi)}(y) \geq 1 \wedge g_{T_{\sigma, \beta}(\psi)}(\infty) / 2\right\} \\
& =\inf \left\{y>0: g_{\psi}(y / \sigma) \geq 1 \wedge g_{\psi}(\infty) / 2\right\}=\sigma s(\psi),
\end{aligned}
$$

showing (6.13). Also the measurability of $s$ can be routinely checked, which verifies that $s$ is a scale function.

Now let $s$ be any scale function. Since the condition $V(\beta)$ holds for $\mathscr{B}$, for every given $\psi_{1}$ in $\mathscr{B}$, the image of the set

$$
\left\{\psi \in \mathscr{B}: \psi=T_{\sigma, \beta}\left(\psi_{1}\right) \text { for some } \sigma>0\right\}
$$

under $s$ is $\mathrm{R}_{+}$. Consequently there exists in the set a unique measure $\nu$ such that $s(\nu)=1$. In fact, this measure is equal to $T_{1 / s(\psi), \beta}(\psi)=T_{s(\psi), \beta}^{-1}(\psi)$ for every $\psi$ in this class, since

$$
s\left(T_{1 / s(\psi), \beta}(\psi)\right)=(1 / s(\psi)) s(\psi)=1 .
$$

Now let $\mathscr{A}$ be the space of such representatives; that is,

$$
\mathscr{A}=\{\psi \in \mathscr{B}: s(\psi)=1\} .
$$

Here $\mathscr{A}$ is equipped with the relative $\sigma$-field. Let $\Pi$ map $(\sigma, \nu) \in \Re_{+} \times \mathscr{A}$ to $T_{\sigma, \beta}(\nu) \in \mathscr{B}$. 
LEMMA 6.6. Assume the notation of Lemma 6.5 in this lemma, and let $\mathscr{A}$ and $\Pi$ be as defined above and $Y$ as in Section 3. Then the mapping

$$
\Upsilon((x, y), \nu)=\Theta(x, \Pi(y, \nu)), \quad((x, y), \nu) \in \mathfrak{R}_{+}^{2} \times \mathscr{A},
$$

is one-one, onto and bimeasurable as a mapping from $\Re_{+}^{2} \times \mathscr{A}$ to its range $\mathcal{S}$ and

$$
\lambda \circ Y=m \times \mathbb{P} \quad \text { on } \Re_{+}^{2} \times \mathscr{A},
$$

where $m$ is Lebesgue measure on $\Re_{+}^{2}$ and $\mathbb{P}$ is a measure on $\mathscr{A}$ satisfying

$$
\int_{\mathscr{A}} \int_{0}^{\infty}\left(1-\exp \left(-\nu(0, t) / t^{\beta}\right)\right) \frac{d t}{t^{2}} \mathbb{P}(d \nu)<\infty .
$$

Proof. First we show that $\Pi$ is one-one and bimeasurable from $\Re_{+} \times \mathscr{A}$ to $\mathscr{B}$. It follows simply from the definition of $\mathscr{A}$ that $\Pi$ is one-one and $\Pi$ is measurable since the mapping

$$
\Omega:(\sigma, \nu) \rightarrow T_{\sigma, \beta}(\nu), \quad \Re_{+} \times \mathscr{M}\left(\Re_{+}\right) \rightarrow \mathscr{M}\left(\Re_{+}\right)
$$

is measurable (in fact continuous) and $\Pi$ is the restriction of $\Omega$ to $\Re_{+} \times \mathscr{A}$. On the other hand, the composition of the following two measurable mappings

$$
\begin{array}{rlrl}
\psi & \rightarrow(s(\psi), \psi), & \mathscr{B} \rightarrow \Re_{+} \times \mathscr{B}, \\
(\sigma, \psi) & \rightarrow\left(\sigma, T_{\sigma, \beta}^{-1}(\psi)\right), \quad \Re_{+} \times \mathscr{B} \rightarrow \Re_{+} \times \mathscr{B}
\end{array}
$$

is $\sigma(\mathscr{B}) \mid \sigma\left(\Re_{+} \times \mathscr{B}\right)$-measurable. Since $\Pi^{-1}$ is in fact this composition and $\mathscr{L}$ has the relative $\sigma$-field, $\Pi^{-1}$ is $\sigma(\mathscr{B}) \mid \sigma\left(\Re_{+} \times \mathscr{A}\right)$-measurable. Thus, as a mapping from $\Re_{+} \times \mathscr{A}$ to $\mathscr{B}, \Pi$ is one-one, onto and bimeasurable. Combining with Lemma 6.5, it is concluded that $Y$ is one-one, onto and bimeasurable as a mapping from $\Re_{+}^{2} \times \mathscr{A}$ to its range $\mathscr{S}$. To show (6.16), in view of (6.7) of Lemma 6.5, it suffices to show that

$$
\mathrm{Q} \circ \Pi=m_{+} \times \mathbb{P} \quad \text { on } \Re_{+} \times \mathscr{A} \text {. }
$$

The approach here is basically the same as that in the proof of (6.10). Fix a measurable set $E$ in $\mathscr{A}$, and define

$$
\xi_{E}(B):=\mathrm{Q} \circ \Pi(B \times E)=\mathrm{Q}\left\{T_{y, \beta}(\nu): y \in B, \nu \in E\right\}, \quad B \in \sigma\left(\Re_{+}\right) .
$$

Then $\xi_{E}$ is a measure on $\sigma\left(\Re_{+}\right)$since $\Pi$ is one-one and bimeasurable. By (6.12), for each $\sigma>0$,

$$
\begin{aligned}
\xi_{E}(\sigma B) & =\mathrm{Q}\left\{T_{\sigma y, \beta}(\nu): y \in B, \nu \in E\right\}=\mathrm{Q}\left\{T_{\sigma, \beta} \circ T_{y, \beta}(\nu): y \in B, \nu \in E\right\} \\
& =\sigma Q\left\{T_{y, \beta}(\nu): y \in B, \nu \in E\right\}=\sigma \xi_{E}(B) .
\end{aligned}
$$

This implies that $\xi_{E}$ can be written as $\mathbb{P}(E) \times m_{+}$where $\mathbb{P}(E)$ is a nonnegative constant and $m_{+}$is Lebesgue measure on $\Re_{+}$. Again, treating $\mathbb{P}$ as a set 
function, it is readily seen that it is a measure on $\mathscr{A}$. Thus (6.18) and hence (6.16) are proved. It remains to show (6.17), which follows from (6.8) by noting

$$
\begin{aligned}
\infty>\int_{\mathscr{B}}(1-\exp (-\psi(0,1))) \mathrm{Q}(d \psi) & =\int_{\mathscr{A}} \int_{0}^{\infty}\left(1-\exp \left(-T_{\sigma, \beta} \circ \nu(0,1)\right)\right) d \sigma \mathbb{P}(d \nu) \\
& =\int_{\mathscr{A}} \int_{0}^{\infty}\left(1-\exp \left(-\nu(0, \sigma) / \sigma^{\beta}\right)\right) \frac{d \sigma}{\sigma^{2}} \mathbb{P}(d \nu) .
\end{aligned}
$$

The proof is complete.

REMARK 2. Note that the arguments in the proof of Lemma 6.6 do not depend on which scale function $s$ we use. In particular, they are not based on the scale function $s$ in (6.15). However, the decomposition (6.16) does depend on the choice of $s$. For clarity of notation, we will denote in the following $\left(\mathscr{A}_{s}, \mathbb{P}_{s}\right)$ the pair of $(\mathscr{A}, \mathbb{P})$ based on a particular scale function $s$.

The proof of the necessity part of Theorem 3.1 is now nearly complete. The only problem is that we have not shown that $\mathbb{P}_{s}$ in (6.16) may be chosen to be a probability measure. For that, our strategy is to first pick an arbitrary scale function $s$, and obtain the pair $\left(\mathscr{A}_{s}, \mathbb{P}_{s}\right)$ according to Lemma 6.6. Then, making use of the information contained in $\mathbb{P}_{s}$, another scale function $s^{\prime}$ is defined from $s$ in such a way that $\mathbb{P}_{s^{\prime}}$ is a probability measure. This is made precise in the following lemma.

Lemma 6.7. There exists a scale function $s$ for $\mathscr{B}$ for which $\mathbb{P}_{s}$ in (6.16) is a finite measure. Furthermore if for some scal efunction $s, \mathbb{P}_{s}\left(\mathscr{A}_{s}\right)=\rho<\infty$, then for any $c>0, \mathbb{P}_{s^{\prime}}\left(\mathscr{A}_{s^{\prime}}\right)=c \rho$ for $s^{\prime}=c^{-1} s$.

Proof. We begin by taking $s$ to be any scale function for $\mathscr{B}$ and show first that $\mathbb{P}_{s}$ is $\sigma$-finite. Since $\mathscr{A}_{s}=\bigcup_{m \geq 1} \bigcup_{n \geq 1}\left\{\nu \in \mathscr{A}_{s}: \nu(0, m)>1 / n\right\}$, it suffices to show that $\mathbb{P}_{s}\left\{\nu \in \mathscr{A}_{s}: \nu(0, a)>\varepsilon\right\}<\infty$ for all $a, \varepsilon>0$. By (6.17),

$$
\begin{aligned}
\infty & >\int_{\mathscr{A}_{s}} \int_{0}^{\infty}\left(1-\exp \left(-\nu(0, \sigma) / \sigma^{\beta}\right)\right) \frac{d \sigma}{\sigma^{2}} \mathbb{P}_{s}(d \nu) \\
& \geq \int_{\left\{\nu \in \mathscr{L}_{s}: \nu(0, a)>\varepsilon\right\}} \int_{a}^{\infty}\left(1-\exp \left(-\nu(0, \sigma) / \sigma^{\beta}\right)\right) \frac{d \sigma}{\sigma^{2}} \mathbb{P}_{s}(d \nu) \\
& \geq \mathbb{P}_{s}\left\{\nu \in \mathscr{A}_{s}: \nu(0, a)>\varepsilon\right\} \int_{a}^{\infty}\left(1-\exp \left(-\varepsilon / \sigma^{\beta}\right)\right) \frac{d \sigma}{\sigma^{2}} .
\end{aligned}
$$

Since $\int_{a}^{\infty}\left(1-\exp \left(-\varepsilon / \sigma^{\beta}\right)\right)\left(d \sigma / \sigma^{2}\right)>0$, we conclude that $\mathbb{P}_{s}\left\{\nu \in \mathscr{A}_{s}: \nu(0, a)>\right.$ $\varepsilon\}<\infty$. Hence $\mathbb{P}_{s}$ is $\sigma$-finite.

Write $\Pi=\Pi_{s}$ and $Y=\Upsilon_{s}$ to stress the dependence of $\Pi, \Upsilon$ on $s$. Let $\left\{E_{i}\right\}$ be a disjoint measurable partition of $\left(\mathscr{A}_{s}, \sigma\left(\mathscr{A}_{s}\right), \mathbb{P}_{s}\right)$ such that $\mathbb{P}_{s}\left(E_{i}\right)<\infty$. This is possible since $\mathbb{P}_{s}$ is $\sigma$-finite. Choose $w_{i}>0$ such that $\sum_{1}^{\infty} w_{i}^{-1} \mathbb{P}_{s}\left(E_{i}\right)=1$. Define

$$
s^{\prime}(\psi)=\sum_{i=1}^{\infty} w_{i} s(\psi) 1\left(\psi \in p_{s}^{-1}\left(E_{i}\right)\right), \quad \psi \in \mathscr{B},
$$


where $p_{s}(\psi)=T_{s(\psi), \beta}^{-1}(\psi)$; namely, $p_{s}$ maps $\psi \in \mathscr{B}$ to its representative in $\mathscr{A}$ ( $p_{s}$ is the second coordinate of $\Pi_{s}^{-1}$ ). Then $s^{\prime}$ is measurable since it is a sum of measurable functions. For each $\sigma>0, \psi \in \mathscr{B}$,

$$
1\left(T_{\sigma, \beta}(\psi) \in p_{s}^{-1}\left(E_{i}\right)\right)=1\left(\psi \in p_{s}^{-1}\left(E_{i}\right)\right)
$$

since if $\psi \in p_{s}^{-1}\left(E_{i}\right)$ then every equivalent $\psi^{\prime}$ is in $p_{s}^{-1}\left(E_{i}\right)$. Thus it is readily seen that $s^{\prime}\left(T_{\sigma, \beta}(\psi)\right)=\sigma s^{\prime}(\psi), \sigma \in \Re_{+}$, and hence $s^{\prime}$ is also a scale function for $\mathscr{B}$. For $\psi \in p_{s}^{-1}\left(E_{i}\right)$,

$$
s^{\prime}(\psi)=w_{i} s(\psi)
$$

and hence if

$$
\nu:=p_{s}(\psi)=T_{s(\psi), \beta}^{-1}(\psi)
$$

then

$$
\nu^{\prime}:=p_{s^{\prime}}(\psi)=T_{s^{\prime}(\psi), \beta}^{-1}(\psi)=T_{w_{i} s(\psi), \beta}^{-1}(\psi)=T_{w_{i}, \beta}^{-1}(\psi) \circ T_{s(\psi), \beta}^{-1}(\psi)=T_{w_{i}, \beta}^{-1}(\nu) .
$$

Thus, for $(x, y) \in \Re_{+}^{2}, \nu \in E_{i}$,

$$
\Upsilon_{s^{\prime}}\left(\left(x, w_{i} y\right), \nu^{\prime}\right)=\Upsilon_{s}((x, y), \nu) .
$$

Hence if $E_{i}^{\prime}=p_{s^{\prime}}\left(p_{s}^{-1}\left(E_{i}\right)\right)$, two applications of (6.16) give

$$
\begin{aligned}
w_{i} \mathbb{P}_{s^{\prime}}\left(E_{i}^{\prime}\right) & =m\left((0,1) \times\left(0, w_{i}\right)\right) \mathbb{P}_{s^{\prime}}\left(E_{i}^{\prime}\right) \\
& =\lambda \circ \Upsilon_{s^{\prime}}\left(\left((0,1) \times\left(0, w_{i}\right)\right) \times E_{i}^{\prime}\right) \\
& =\lambda \circ \Upsilon_{s}\left(((0,1) \times(0,1)) \times E_{i}\right) \\
& =m((0,1) \times(0,1)) \mathbb{P}_{s}\left(E_{i}\right)=\mathbb{P}_{s}\left(E_{i}\right) .
\end{aligned}
$$

Since, by the proof of Lemma $6.6, \Pi_{s}$ is bimeasurable, it follows that

$$
\Pi_{s^{\prime}}^{-1}\left(\Pi_{s}\left(\Re_{+} \times E_{i}\right)\right)=\Re_{+} \times p_{s^{\prime}}\left(p_{s}^{-1}\left(E_{i}\right)\right)
$$

is measurable in $\Re_{+} \times \mathscr{A}_{s^{\prime}}$. Thus $E_{i}^{\prime}=p_{s^{\prime}}\left(p_{s}^{-1}\left(E_{i}\right)\right)$ is measurable in $\mathscr{A}_{s}$ and it is clear that $\left\{E_{i}^{\prime}=p_{s^{\prime}}\left(p_{s}^{-1}\left(E_{i}\right)\right)\right\}$ is a disjoint measurable partition of $\mathscr{A}_{s^{\prime}}$. Therefore by the choice of $w_{i}$,

$$
\mathbb{P}_{s^{\prime}}\left(\mathscr{A}_{s^{\prime}}\right)=\sum_{i=1}^{\infty} \mathbb{P}_{s^{\prime}}\left(E_{i}^{\prime}\right)=\sum_{i=1}^{\infty} w_{i}^{-1} \mathbb{P}_{s}\left(E_{i}\right)=1,
$$

which shows that $\mathbb{P}_{s^{\prime}}$ is a probability measure. The second claim also follows in an obvious way from the above proof. 
6.3. The proof of Theorem 3.2. The proof is Iargely the same as that of Theorem 3.1, with the following minor modifications. First, as before, we only show the necessity part. Thus it is assumed throughout that conditions (A1), (A2), $\left(A 3^{\prime}\right)$ and $(A 4)$ hold. First we note that $\eta_{d}$ is absent by $\left(A 3^{\prime}\right)$.

Lemma 6.8. The set $\mathscr{B}$ of Lemma 6.5 is a subset of $\left\{\psi \in \mathscr{M}\left(\Re_{+}\right) \backslash\{0\}\right.$ : $\psi(0, \varepsilon)=0$ for some $\varepsilon>0\}$.

Proof. First define

$$
\Lambda=\Theta\left(\Re \times\left\{\psi \in \mathscr{M}\left(\Re_{+}\right) \backslash\{0\}: \psi(0, \varepsilon)=0 \text { for some } \varepsilon>0\right\}\right) .
$$

It suffices to show that $\lambda\left(\Lambda^{c}\right)=0$ and the rest of the proof completely parallels that of Lemma 6.5. Write

$$
\Lambda=A \cap B,
$$

where $A$ is as defined in the proof of Lemma 6.5 , and

$$
B=\left\{\mu \in \mathscr{M}\left(\Re_{+}^{2}\right) \backslash\{0\}: \mu(\Re \times(0, \varepsilon))=0 \text { for some } \varepsilon>0\right\} .
$$

It is easy to see that

$$
A \cap B^{c} \subset \bigcup_{m=1}^{\infty} \bigcap_{n=1}^{\infty}\left\{\mu \in \mathscr{M}\left(\Re_{+}^{2}\right) \backslash\{0\}: \mu\left([-m, m) \times\left(0, \frac{1}{n}\right)\right)>0\right\}
$$

and hence by (6.9),

$$
\begin{aligned}
\lambda\left(A \cap B_{1}^{c}\right) & \leq \lim _{m \rightarrow \infty} \lim _{n \rightarrow \infty} \lambda\left\{\mu \in \mathscr{M}\left(\Re_{+}^{2}\right) \backslash\{0\}: \mu\left([-m, m) \times\left(0, \frac{1}{n}\right)\right)>0\right\} \\
& \leq \lim _{m \rightarrow \infty} \lim _{n \rightarrow \infty} 2 m \lambda\left\{\mu \in \mathscr{M}\left(\Re_{+}^{2}\right) \backslash\{0\}: \mu\left([0,1) \times\left(0, \frac{1}{n}\right)\right)>0\right\},
\end{aligned}
$$

where the right-hand side is 0 by (ii) of Lemma 6.4. Thus we have shown that $\lambda\left(\Lambda^{c}\right)=0$, which concludes the proof.

Note that by Lemma 6.4, $\rho:=-\log P\{\eta([0,1) \times(0,1))=0\} \in(0, \infty)$. For the set $\mathscr{B}$ in Lemma 6.8, define the function $s$ by

$$
s: \psi \rightarrow \inf \{y>0: \psi(0, y / \rho)>0\}, \quad \mathscr{B} \rightarrow \Re_{+} .
$$

It is easily verified that $s$ is a scale function for $\mathscr{B}$. As before, define the space $\mathscr{A}=\{\psi \in \mathscr{B}: s(\psi)=1\}$. The proof of Theorem 3.2 is complete once it is shown that the measure $\mathbb{P}$ in (6.16) based on this scale function is a probability measure. Observe that $\mathscr{A}$ contains measures in $\mathscr{M}\left(\Re_{+}\right)$whose supports start at $\rho^{-1}$. Thus,

$$
\Upsilon(((0,1) \times(0, \rho)) \times \mathscr{A})=\{\phi \in \Lambda: \phi((0,1) \times(0,1))>0\},
$$


where $\Lambda$ is as defined in the proof of Lemma 6.8. This implies that

$$
\begin{aligned}
\mathbb{P}(\mathscr{A}) & =\frac{\lambda\{\phi \in \Lambda: \phi((0,1) \times(0,1))>0\}}{m((0,1) \times(0, \rho))} \\
& =-\log P\{\eta((0,1) \times(0,1))=0\} / \rho=1,
\end{aligned}
$$

which completes the proof.

6.4. The proof of Proposition 3.3. By Remark 2 of Section 3, it suffices to show that the Laplace transform of $\sum Y\left(\left(X_{i}, Y_{i}\right), \gamma_{i}\right)$ is equal to

$$
\begin{aligned}
\exp \left\{-\int_{\mathscr{A}} \int_{\mathfrak{R}_{+}^{2}}\left(1-\exp \left(-\int_{0}^{\infty} f(x, y z) y^{\beta} \nu(d z)\right)\right) m(d x d y) \mathbb{P}(d \nu)\right\}, & \\
& f \in \mathscr{F}\left(\Re_{+}^{2}\right) .
\end{aligned}
$$

Let $E_{q, r}=(q-1, q] \times(r-1, r]$ and

$$
\eta_{q, r}=\sum_{\left(X_{i}, Y_{i}\right) \in E_{q, r}} \Upsilon\left(\left(X_{i}, Y_{i}\right), \gamma_{i}\right), \quad r \geq 0, q, r \in \mathrm{I},
$$

where I is the set of all integers. Conditional on $\Gamma\left(E_{q, r}\right)=k$, where $k$ is any nonnegative integer, the points of $\Gamma$ in $E_{q, r}$ are independently and uniformly distributed over $E_{q, r}$. Thus for any $f \in \mathscr{T}\left(\Re_{+}^{2}\right)$,

$$
\begin{aligned}
\mathscr{E} \exp & \left\{-\int_{\mathfrak{R}_{+}^{2}} f d \eta_{q, r}\right\} \\
= & \sum_{k=0}^{\infty} P\left\{\Gamma\left(E_{q, r}\right)=k\right\} \mathscr{E}\left\{\exp \left(-\int_{\mathfrak{R}_{+}^{2}} f d \eta_{q, r}\right) \mid \Gamma\left(E_{q, r}\right)=k\right\} \\
= & \sum_{k=0}^{\infty} \frac{\exp (-1)}{k !}\left\{\int_{\mathscr{A}} \int_{E_{q, r}} \exp \left(-\int_{0}^{\infty} f(x, y z) y^{\beta} \nu(d z)\right) d x d y \mathbb{P}(d \nu)\right\}^{k} \\
= & \exp \left\{-\int_{\mathscr{A}} \int_{E_{q, r}}\left(1-\exp \left(-\int_{0}^{\infty} f(x, y z) y^{\beta} \nu(d z)\right) d x d y \mathbb{P}(d \nu)\right\} .\right.
\end{aligned}
$$

The result follows from the fact that $\sum Y\left(\left(X_{i}, Y_{i}\right), \gamma_{i}\right)$ can be written as the sum of the random measures $\eta_{q, r}, r \geq 0, q, r \in \mathrm{I}$, which are mutually independent.

6.5. The proof of Theorem 4.1. For each $B \in \sigma\left(\Re_{+}\right)$, let

$$
\begin{array}{r}
\mathscr{M}_{B}=\left\{\psi \in \mathscr{M}\left(\Re_{+}\right): \psi(0, y)=0 \text { for some } y>0 \text { (depending on } \psi\right) \text { and } \\
\psi(B)>0\} .
\end{array}
$$

Also define $\mathscr{F}_{\text {step }}\left(\Re_{+}\right)$as the collection of step functions $f$ of the form

$$
f(y)=\sum_{i=1}^{k} s_{i} I_{\left(0, \tau_{i}\right)}(y), \quad y>0,
$$


where $k \geq 1, s_{i} \geq 0,0<\tau_{i}<\infty$. We start by stating some conclusions that will be used repeatedly throughout the proof. First define

$$
\omega_{T}(\cdot)=a_{T} \int_{0}^{r_{T}} I\left(u_{T}^{-1}\left(\xi_{t}\right) \in \cdot\right) d t
$$

and

$$
\mathrm{Q}_{T}=k_{T} P \circ \omega_{T}^{-1} .
$$

Routine calculations using the mixing condition and Lemma 6.1 show that

$$
\begin{aligned}
-\log & E \exp \left\{-\int_{\Re_{+}^{2}} I_{[0,1)}(x) f(y) d \eta_{T}(x, y)\right\} \\
& =k_{T} E\left(1-\exp \left(-\omega_{T} f\right)\right)+o(1) \\
& =\int(1-\exp (-\psi f)) \mathrm{Q}_{T}(d \psi)+o(1), \quad f \in \mathscr{F}_{\text {step }}\left(\Re_{+}\right) .
\end{aligned}
$$

Also using the mixing condition and the assumption $P\left\{M(T) \leq u_{T}(\tau)\right\} \rightarrow e^{-\tau}$, it follows analogously to Lemma 2.3 of Hsing, Hüsler and Leadbetter (1988) that

$$
\mathrm{Q}_{T}\left(\mathscr{M}_{(0, \tau)}\right)=k_{T} P\left\{\eta_{T}([0,1) \times(0, \tau))>0\right\} \rightarrow \tau, \quad \tau>0 .
$$

We first prove that $\eta_{T}$ converges in distribution to a nontrivial random measure if there exists a measure $Q$ on $\mathscr{M}_{(0, \infty)}$ such that

$$
\begin{aligned}
\lim _{T \rightarrow \infty} k_{T} E\left(1-\exp \left(-\omega_{T} f\right)\right)=\int_{\mathscr{M}_{(0, \infty)}}(1-\exp (-\psi f)) \mathrm{Q}(d \psi) & <\infty, \\
& f \in \mathscr{F}_{\text {step }}\left(\Re_{+}\right) .
\end{aligned}
$$

With the latter assumption, use of (6.20) shows that the random vectors $\eta_{T}\left([0,1) \times\left(0, \tau_{1}\right)\right), \ldots, \eta_{T}\left([0,1) \times\left(0, \tau_{k}\right)\right)$ jointly converge in distribution for all $\tau_{1}, \ldots, \tau_{k}>0$. It may be shown in the same manner as in Theorem 3.2 of Leadbetter and Hsing (1990) that this implies that the random measures

$$
\eta_{T}\left((\cdot \cap[0,1)) \times\left(0, \tau_{1}\right)\right), \ldots, \eta_{T}\left((\cdot \cap[0,1)) \times\left(0, \tau_{k}\right)\right)
$$

jointly converge in distribution. In particular this implies that for any $\left[a_{i}, b_{i}\right) \subset[0,1), 1 \leq i \leq k$,

$$
\eta_{T}\left(\left[a_{1}, b_{1}\right) \times\left(0, \tau_{1}\right)\right), \ldots, \eta_{T}\left(\left[a_{k}, b_{k}\right) \times\left(0, \tau_{k}\right)\right)
$$

jointly converge in distribution. It is further possible to show using stationarity and the property (U2) (which holds by the mixing condition and the condition on $u_{T}$ ) that the restriction of $\left[a_{i}, b_{i}\right) \subset[0,1)$ can be relaxed. Hence, by a standard existence criterion [see, for example, Lemma 5.1 of Kallenberg (1983)], $\eta_{T}$ converges in distribution to a nontrivial random measure. In view of this, to prove Theorem 4.1 it suffices to show that the assumptions of the theorem imply (6.22) for some Q. Similar to Section 6.2, let $\Pi$ map $(\sigma, \nu) \in \Re_{+} \times \tilde{\mathscr{M}}$ 
to $T_{\sigma, \beta}(\nu) \in \mathscr{M}_{(0, \infty)}$. It is clear that $\Pi$ is one-one and bicontinuous. For any $y>0$,

$$
\begin{aligned}
\frac{\mathrm{Q}_{T}\left(\Pi((0, y) \times \cdot) \cap \mathscr{M}_{(0, \tau)}\right)}{\mathrm{Q}_{T}\left(\mathscr{M}_{(0, \tau)}\right)} & =\frac{\mathrm{Q}_{T} \circ \Pi((0, y \wedge \tau) \times \cdot)}{\mathrm{Q}_{T}\left(\mathscr{M}_{(0, \tau)}\right)} \\
& =\frac{\mathrm{Q}_{T} \circ \Pi((0, y \wedge \tau) \times \cdot)}{\mathrm{Q}_{T}\left(\mathscr{M}_{(0, y \wedge \tau)}\right)} \frac{\mathrm{Q}_{T}\left(\mathscr{M}_{(0, y \wedge \tau)}\right)}{\mathrm{Q}_{T}\left(\mathscr{M}_{(0, \tau)}\right)} \\
& \rightarrow{ }_{w} \mathbb{P}(\cdot) \frac{y \wedge \tau}{\tau} \text { on } \tilde{\mathscr{M}},
\end{aligned}
$$

where the weak convergence follows using the assumption and the fact that the first ratio is simply $P\left\{\chi_{T} \in \cdot \mid M\left(r_{T}\right)>u_{T}(y \wedge \tau)\right\}$. This implies that

$$
\frac{\mathrm{Q}_{T}\left(\Pi(\cdot \times \cdot) \cap \mathscr{M}_{(0, \tau)}\right)}{\mathrm{Q}_{T}\left(\mathscr{M}_{(0, \tau)}\right)} \rightarrow_{w} \frac{m_{+}(\cdot \cap(0, \tau)) \times \mathbb{P}(\cdot)}{m_{+}(0, \tau)} \text { on }(0, \infty) \times \tilde{\mathscr{M}},
$$

which implies, by the fact that $\Pi$ is bicontinuous, that

$$
\frac{\mathrm{Q}_{T}\left(\cdot \cap \mathscr{M}_{(0, \tau)}\right)}{\mathrm{Q}_{T}\left(\mathscr{M}_{(0, \tau)}\right)} \rightarrow{ }_{w} \frac{\left(m_{+} \times \mathbb{P}\right) \circ \Pi^{-1}\left(\cdot \cap \mathscr{M}_{(0, \tau)}\right)}{\tau} \text { on } \mathscr{M}_{(0, \infty)} .
$$

Defining $\mathrm{Q}=\left(m_{+} \times \mathbb{P}\right) \circ \Pi^{-1}$, the last convergence can be rewritten as

$$
\frac{\mathrm{Q}_{T}\left(\cdot \cap \mathscr{M}_{(0, \tau)}\right)}{\mathrm{Q}_{T}\left(\mathscr{M}_{(0, \tau)}\right)} \rightarrow{ }_{w} \frac{\mathrm{Q}\left(\cdot \cap \mathscr{M}_{(0, \tau)}\right)}{\mathrm{Q}\left(\mathscr{M}_{(0, \tau)}\right)} \text { on } \mathscr{M}_{(0, \infty)},
$$

which implies that for any $f \in \mathscr{F}_{\text {step }}\left(\Re_{+}\right)$with support in $(0, \tau)$,

$$
\frac{\int_{\mathscr{M}_{(0, \tau)}} e^{-\psi f} \mathrm{Q}_{T}(d \psi)}{\mathrm{Q}_{T}\left(\mathscr{M}_{(0, \tau)}\right)} \rightarrow \frac{\int_{\mathscr{M}_{(0, \tau)}} e^{-\psi f} \mathrm{Q}(d \psi)}{\mathrm{Q}\left(\mathscr{M}_{(0, \tau)}\right)} .
$$

Since $\mathrm{Q}_{T}\left(\mathscr{M}_{(0, \tau)}\right) \rightarrow \mathrm{Q}\left(\mathscr{M}_{(0, \tau)}\right)$ by (6.21), and the support of $f$ is contained in $(0, \tau)$, this implies that

$$
\int\left(1-e^{-\psi f}\right) \mathrm{Q}_{T}(d \psi) \rightarrow \int\left(1-e^{-\psi f}\right) \mathrm{Q}(d \psi) .
$$

Since this holds for all $\tau>0$ and $f \in \mathscr{T}_{\text {step }}\left(\Re_{+}\right),(6.22)$ is proved.

\subsection{Proofs for Section 5 .}

Proof of Proposition 5.1. In this proof we do not directly use Theorem 4.1, but rather the essence of it. Note that since $\left\{\bar{\xi}_{t}, i \leq t<i+1\right\}, i \in \mathrm{I}$, are i.i.d., it suffices to show that conditional on $\sup _{0 \leq s<1} \bar{\xi}_{s}>u_{T}(\tau)$,

$$
\begin{aligned}
& T^{\beta}\left(u_{T}^{-1}\left(\sup _{0 \leq s<1} \bar{\xi}_{s}\right)\right)^{-\beta} \int_{0}^{1} I\left(\frac{u_{T}^{-1}\left(\bar{\xi}_{t}\right)}{u_{T}^{-1}\left(\sup _{0 \leq s<1} \bar{\xi}_{s}\right)} \leq x\right) d t \\
& \quad \rightarrow \log x, \quad x \geq 1
\end{aligned}
$$


for each $\tau>0$. Notice that $\sup _{0 \leq s<1} \bar{\xi}_{s}=X_{0}$ and

$$
\frac{u_{T}^{-1}\left(\bar{\xi}_{t}\right)}{u_{T}^{-1}\left(\sup _{0 \leq s<1} \bar{\xi}_{s}\right)}=\frac{T \exp \left(-\left(X_{0}-t \exp \left(\beta X_{0}\right)\right)\right.}{T e^{-X_{0}}}=\exp \left(t \exp \left(\beta X_{0}\right)\right) .
$$

Thus

$$
\begin{gathered}
T^{\beta}\left(u_{T}^{-1}\left(\sup _{0 \leq s<1} \bar{\xi}_{s}\right)\right)^{-\beta} \int_{0}^{1} I\left(\frac{u_{T}^{-1}\left(\bar{\xi}_{t}\right)}{u_{T}^{-1}\left(\sup _{0 \leq s<1} \bar{\xi}_{s}\right)} \leq x\right) d t \\
=T^{\beta}\left(T \exp \left(-X_{0}\right)\right)^{-\beta} \int_{0}^{1} I\left(\exp \left(t e^{\beta X_{0}}\right) \leq x\right) d t \\
=T^{\beta}\left(T \exp \left(-X_{0}\right)\right)^{-\beta} \int_{0}^{1} I\left(t \leq \frac{\log x}{\exp \left(\beta X_{0}\right)}\right) d t,
\end{gathered}
$$

which, if $X_{0}$ is large, is equal to

$$
T^{\beta}\left(T \exp \left(-X_{0}\right)\right)^{-\beta} \log x / \exp \left(\beta X_{0}\right)=\log x .
$$

This establishes (6.23) and completes the proof.

A SKETCHED PROOF OF PROPOSITION 5.2. We only provide an explanation of why the result is true, referring the reader to Hsing and Leadbetter (1991) for a complete proof. First, note that we can assume without loss of generality that $a$ has compact support so that $\left\{\xi_{t}\right\}$ is $m$-dependent. For, if this is not the case, writing

$$
a^{(m)}(t)=a(t) I(|t| \leq m / 2)
$$

and

$$
\xi_{t}^{(m)}=\int a^{(m)}(t-x) d Z(x), \quad-\infty<t<\infty,
$$

it is easy to show that

$$
\lim _{m \rightarrow \infty} \lim _{T \rightarrow \infty} P\left\{\sup _{0 \leq t \leq T}\left|\xi_{t}-\xi_{t}^{(m)}\right|>u_{T}(\tau)\right\}=0, \quad \tau>0 .
$$

Hence a standard uniform convergence argument combined with the following will suffice. Also for notational convenience we assume that $a(t)$ is nonnegative. By $m$-dependence and Theorem 4.1, it is enough to show that there exists a sequence $k_{T}$ such that $k_{T} \rightarrow \infty, r_{T}:=T / k_{T} \rightarrow \infty$ and

$$
\begin{aligned}
& \lim _{T \rightarrow \infty} P\left\{\int_{0}^{r_{T}} I\left(\frac{u_{T}^{-1}\left(\xi_{t}\right)}{u_{T}^{-1}\left(M\left(r_{T}\right)\right)} \leq x\right) d t>z \mid M\left(r_{T}\right)>u_{T}(\tau)\right\} \\
& \quad=I\left\{\int_{-\infty}^{\infty} I\left(a(t) \geq A x^{-1 / \alpha}\right) d t>z\right\}, \quad x \geq 1, z>0, \tau>0 .
\end{aligned}
$$

Let $x \geq 1, z>0, \tau>0$ be fixed from now on. As in Rootzén (1978), let $h(T)>0$ be such that $h(T) \rightarrow \infty$ and $h(T) / T \rightarrow 0$ but otherwise arbitrary, and let

$$
t_{T 1}=\inf \left\{t \geq h(T): \xi_{t}>u_{T}(\tau)\right\} \text {. }
$$


By Theorem 5.2 of Rootzén (1978), there is a sequence of constants $\left\{\tau_{T 1}\right\}$ such that

$$
\lim _{\delta \rightarrow \infty} \limsup _{T \rightarrow \infty} P\left\{\left|t_{T 1}-\tau_{T 1}\right|>\delta\right\}=0
$$

and as $T \rightarrow \infty$,

$$
P\left\{Y_{T 1}(t) / u_{T}(\tau) \in \cdot \mid t_{T 1} \leq \varepsilon T\right\} \rightarrow_{w} P\{Z a(-t) \in \cdot\} \quad \text { on } D(-\infty, \infty), \varepsilon>0,
$$

where $Y_{T 1}(t)=\xi_{t+\tau_{T 1}}$ and

$$
P\{Z \leq z\}=1-A^{-\alpha}(1+\gamma) z^{-\alpha}, \quad z \geq A(1+\gamma)^{1 / \alpha} .
$$

Thus there exists $k_{T}$ (which can be chosen independent of $\tau>0$ ) such that $k_{T} \rightarrow \infty, r_{T}:=T / k_{T} \rightarrow \infty, h(T) / r_{T} \rightarrow 0$, and

$$
P\left\{Y_{T 1}(t) / u_{T}(\tau) \in \cdot \mid t_{T 1} \leq r_{T}\right\} \rightarrow{ }_{w} P\{Z a(-t) \in \cdot\} \quad \text { on } D(-\infty, \infty) .
$$

It turns out that the two events $\left(M\left(r_{T}\right)>u_{T}(\tau)\right)$ and $\left(t_{T_{1}}<r_{T}\right)$ approximate each other so that

$$
\begin{aligned}
& P\left\{\int_{0}^{r_{T}} I\left(\frac{u_{T}^{-1}\left(\xi_{t}\right)}{u_{T}^{-1}\left(M\left(r_{T}\right)\right)} \leq x\right) d t>z \mid M\left(r_{T}\right)>u_{T}(\tau)\right\} \\
& \quad=P\left\{\int_{-m}^{m} I\left(Y_{T 1}(t) \geq x^{-1 / \alpha} \sup _{-m<s<m} Y_{T 1}(s)\right) d t>z \mid t_{T 1}<r_{T}\right\}+o(1),
\end{aligned}
$$

the proof of which can be found in Hsing and Leadbetter (1991). Now (6.24) follows by virtue of (6.25).

A SKetched PRoOF of Proposition 5.3. Again, some technical details will be omitted to save space. A detailed proof is contained in Hsing and Leadbetter (1991). Since $\left\{\xi_{t}\right\}$ is strongly mixing, the sequence $k_{T}$ in Theorem 4.1 exists for $\left\{\xi_{t}\right\}$. By Theorem 4.1 and the fact that $\mathbb{P}\{\nu\}=1, \nu$ being defined in Proposition 5.3, it suffices to show that

$$
\begin{gathered}
P\left\{a_{T} \int_{0}^{r_{T}} I\left(\frac{u_{T}^{-1}\left(\xi_{t}\right)}{u_{T}^{-1}\left(M\left(r_{T}\right)\right)} \leq y\right) d t \leq x \mid M\left(r_{T}\right)>u_{T}(\tau)\right\} \\
\rightarrow I(\nu[1, y] \leq x), \quad \tau, x>0, y \geq 1,
\end{gathered}
$$

which, after replacing $u_{T}^{-1}(u)$ by the expression given in (5.3.1), becomes

$$
\begin{aligned}
P_{T} & :=P\left\{a_{T} \int_{0}^{r_{T}} I\left(\xi_{t} \geq\left(M^{2}\left(r_{T}\right)-2 \log y\right)^{1 / 2}\right) d t \leq x \mid M\left(r_{T}\right)>u_{T}(\tau)\right\} \\
& \rightarrow I(\nu[1, y] \leq x), \quad \tau,>0, y \geq 1 .
\end{aligned}
$$

Then (6.26) is proved using the idea that the behavior of a normal process at a high-level crossing can be described by a Slepian model process, defined (for $u \in \Re$ ) by

$$
\xi_{u, t}=u r(t)-\frac{\zeta r^{\prime}(t)}{\lambda_{2}}+\kappa_{t}, \quad t \in \Re
$$


where $\zeta$ has density $\left(z / \lambda_{2}\right) \exp \left(-z^{2} / 2 \lambda_{2}\right), z>0$, and $\kappa_{t}$ is a nonstationary normal process, independent of $\zeta$ with zero mean, and with the covariance function

$$
\operatorname{cov}\left(\kappa_{s}, \kappa_{t}\right)=r(s-t)-r(s) r(t)-\frac{r^{\prime}(s) r^{\prime}(t)}{\lambda_{2}} .
$$

See Chapter 10 of Leadbetter, Lindgren and Rootzén (1983) for details. Let $\hat{\xi}_{u}$ be the supremum of $\xi_{u, t}$ for $t$ between 0 and the first time a downcrossing of $u$ by $\xi_{u, t}$ occurs (cf. Corollary 10.3.3 of Leadbetter, Lindgren and Rootzén (1983). Also define

$$
\xi_{\infty, t}=-\frac{\lambda_{2} t^{2}}{2}+\zeta t, \quad t \in \Re
$$

It follows from Theorem 10.4.2 of Leadbetter, Lindgren and Rootzén (1983) that $\xi_{\infty, t}$ is the a.s. limit of $u\left(\xi_{u, t / u}-u\right)$ as $u \rightarrow \infty$. Let $\hat{\xi}_{\infty}$ be the global supremum of $\xi_{\infty, t}$. Also for $\delta>0$, define

$$
Q_{T}(\delta)=P\left\{u_{T}(1) \int_{-\delta / u_{T}(\tau)}^{\delta / u_{T}(\tau)} I\left(\xi_{u_{T}(\tau), t} \geq\left(\hat{\xi}_{u_{T}(\tau)}^{2}-2 \log y\right)^{1 / 2}\right) d t \leq x\right\} .
$$

It can be shown that

$$
\lim _{\delta \rightarrow \infty} \lim _{T \rightarrow \infty}\left|P_{T}-Q_{T}(\delta)\right|=0
$$

so that it suffices to analyze $Q_{T}(\delta)$. In the integral of the definition of $Q_{T}(\delta)$, making a change-of-variables with $v=u_{T}(\tau) t$,

$$
\begin{aligned}
Q_{T}(\delta)=P\left\{\int _ { - \delta } ^ { \delta } I \left(\xi_{u_{T}(\tau), v / u_{T}(\tau)} \geq\left(\hat{\xi}_{u_{T}(\tau)}^{2}\right.\right.\right. & \left.-2 \log y)^{1 / 2}\right) d v \\
& \left.\leq x u_{T}(\tau) / u_{T}(1)\right\} .
\end{aligned}
$$

By Theorem 10.4.2 of Leadbetter, Lindgren and Rootzén (1983),

$$
u_{T}(\tau)\left(\xi_{u_{T}(\tau), v / u_{T}(\tau)}-u_{T}(\tau)\right) \rightarrow \xi_{\infty, v} \quad \text { a.s. uniformly for }|v| \leq \delta
$$

for any finite $\delta$. Thus

$$
\begin{aligned}
\hat{\xi}_{u_{T}(\tau)}^{2}-\xi_{u_{T}(\tau), v / u_{T}(\tau)}^{2}= & \left(\hat{\xi}_{u_{T}(\tau)}-u_{T}(\tau)\right)^{2}-\left(\xi_{u_{T}(\tau), v / u_{T}(\tau)}-u_{T}(\tau)\right)^{2} \\
& +2 u_{T}(\tau)\left(\hat{\xi}_{u_{T}(\tau)}-u_{T}(\tau)\right) \\
& -2 u_{T}(\tau)\left(\xi_{u_{T}(\tau), v / u_{T}(\tau)}-u_{T}(\tau)\right) \\
\rightarrow & 2\left(\hat{\xi}_{\infty}-\xi_{\infty, v}\right) \quad \text { a.s. uniformly for }|v| \leq \delta .
\end{aligned}
$$

Therefore, since $u_{T}(\tau) / u_{T}(1) \rightarrow 1$,

$$
\begin{gathered}
P\left\{\int_{-\delta}^{\delta} I\left(\xi_{u_{T}(\tau), v / u_{T}(\tau)} \geq\left(\hat{\xi}_{u_{T}(\tau)}^{2}-2 \log y\right)^{1 / 2}\right) d v \leq x u_{T}(\tau) / u_{T}(1)\right\} \\
\rightarrow P\left(\int_{-\delta}^{\delta} I\left(2\left(\hat{\xi}_{\infty}-\xi_{\infty, v}\right) \leq 2 \log y\right) d v \leq x\right), \quad \text { as } T \rightarrow \infty
\end{gathered}
$$


which converges, as $\delta \rightarrow 0$, to

$$
\begin{gathered}
P\left(\int_{-\infty}^{\infty} I\left(2\left(\hat{\xi}_{\infty}-\xi_{\infty, v}\right) \leq 2 \log y\right) d v \leq x\right) \\
\quad=I\left(\int_{-\infty}^{\infty} I\left(\lambda_{2} v^{2} \leq 2 \log y\right) d v \leq x\right) \\
\quad=I\left(2\left(\lambda_{2}^{-1} 2 \log y\right)^{1 / 2} \leq x\right) .
\end{gathered}
$$

Thus the result follows from (6.27)-(6.30).

Proof of Proposition 5.4. First, let $0<x<1$. It follows from (5.2) and Lemma 6.1 that for $s>0$,

$$
\begin{aligned}
& -\log E\left(\exp \left\{-s a_{T} \zeta_{T}([0, x) \times(0, \tau))\right\}\right) \\
& \quad=x k_{T} E\left(1-\exp \left\{-s a_{T} \zeta_{T}\left(\left[0, T / k_{T}\right) \times(0, \tau)\right)\right\}\right)+o(1) \\
& \quad=x k_{T} E\left(1-\exp \left\{-s a_{T} \int_{0}^{T / k_{T}} I\left(\xi_{t}>u_{T}(\tau)\right) d t\right\}\right)+o(1) .
\end{aligned}
$$

By Bonferroni's inequality,

$$
\begin{aligned}
& E\left(s a_{T} \int_{0}^{T / k_{T}} I\left(\xi_{t}>u_{T}(\tau)\right) d t\right)-\frac{1}{2} E\left(s a_{T} \int_{0}^{T / k_{T}} I\left(\xi_{t}>u_{T}(\tau)\right) d t\right)^{2} \\
& \quad \leq E\left(1-\exp \left\{-s a_{T} \int_{0}^{T / k_{T}} I\left(\xi_{t}>u_{T}(\tau)\right) d t\right\}\right) \\
& \quad \leq E\left(s a_{T} \int_{0}^{T / k_{T}} I\left(\xi_{t}>u_{T}(\tau)\right) d t\right) .
\end{aligned}
$$

By stationarity,

$$
E\left(s a_{T} \int_{0}^{T / k_{T}} I\left(\xi_{t}>u_{T}(\tau)\right) d t\right)=\frac{s a_{T} T}{k_{T}} P\left\{\xi_{0}>u_{T}(\tau)\right\} \sim \frac{\tau^{\beta+1} s}{k_{T}} .
$$

Also

$$
\begin{aligned}
E\left(s a_{T} \int_{0}^{T / k_{T}} I\left(\xi_{t}>u_{T}(\tau)\right) d t\right)^{2} \\
\quad \leq \frac{s^{2} a_{T}^{2} T}{k_{T}}\left(\int_{0}^{l_{T}}+\int_{0}^{T / k_{T}}\right) P\left\{\xi_{0}>u_{T}, \xi_{t}>u_{T}(\tau)\right\} d t \\
\quad \leq \frac{s^{2} a_{T}^{2} T}{k_{T}}\left(l_{T} P\left\{\xi_{0}>u_{T}(\tau)\right\}+\frac{T}{k_{T}} \alpha_{T, l_{T}}(\tau)+\frac{T}{k_{T}} P^{2}\left\{\xi_{0}>u_{T}(\tau)\right\}\right) \\
\quad=\frac{s^{2} a_{T} l_{T}}{k_{T}}\left(\tau^{\beta+1}+o(1)\right)+\frac{s^{2} a_{T}^{2} T^{2}}{k_{T}^{2}} \alpha_{T, l_{T}}(\tau)+\frac{s^{2}}{k_{T}^{2}}\left(\tau^{2(\beta+1)}+o(1)\right) .
\end{aligned}
$$


By (5.2),

$$
\begin{aligned}
\limsup _{n \rightarrow \infty} k_{T} \frac{a_{T} l_{T}}{k_{T}} & \leq \limsup _{n \rightarrow \infty} \frac{a_{T} T}{k_{T}} \lim _{n \rightarrow \infty} \frac{k_{T} l_{T}}{T}=0, \\
\limsup _{n \rightarrow \infty} k_{T} \frac{a_{T}^{2} T^{2}}{k_{T}^{2}} \alpha_{T, l_{T}}(\tau) & \leq \limsup _{n \rightarrow \infty} \frac{a_{T}^{2} T^{2}}{k_{T}^{2}} \lim _{n \rightarrow \infty} k_{T} \alpha_{T, l_{T}}(\tau)=0 .
\end{aligned}
$$

Hence,

$$
x k_{T} E\left(1-\exp \left\{-s a_{T} \int_{0}^{T / k_{T}} I\left(\xi_{t}>u_{T}(\tau)\right) d t\right\}\right) \rightarrow x \tau^{\beta+1} s
$$

Thus

$$
\lim _{T \rightarrow \infty} E\left(\exp \left\{-s a_{T} \zeta_{T}([0, x) \times(0, \tau))\right\}\right)=\exp \left(-x \tau^{\beta+1} s\right)
$$

Since the limit is degenerate, the result follows readily.

\section{REFERENCES}

Berman, S. (1980). A compound Poisson limit for stationary sums and sojourns of Gaussian processes. Ann. Probab. 8 511-538.

BERMAN, S. (1982). Sojourns and extremes of stationary processes. Ann. Probab. 10 1-46.

BERMAN, S. (1983). Sojourns of stationary processes in rare sets. Ann. Probab. 11 847-866.

Billingsley, P. (1968). Convenience of Probability Measures. Wiley, New York.

Cramér, H. and LeAdbetTer, M. R. (1967). Stationary and Related Stochastic Processes. Wiley, New York.

HsING, T. (1987). On the characterization of certain point processes. Stochastic Process. Appl. 26 297-316.

Hsing, T. (1993). On some estimates based on sample behavior near high-level excursions. Probab. Theory Related Fields 95 331-356.

Hsing, T., HÜsleR, J. and LEAdBETTER, M. R. (1988). On the exceedance point process for a stationary sequence. Probab. Theory Related Fields 78 97-112.

Hsing, T. and LEADBETTER, M. R. (1997). On multiple-level excursions by stationary processes with deterministic peaks. Stochastic Process. Appl. 71 11-32.

Ibragimov, I. A. and Rozanov, Y. A. (1978). Gaussian Random Processes. Springer, New York.

Kallenberg, O. (1983). Random Measures. Academic Press, New York.

LEADBETTER, M. R. (1983). Extremes and local dependence in a stationary sequence. Z. Wahrsch. Verw. Gebiete 65 291-306.

LEADBETteR, M. R. and Hsing, T. (1990). Limit theorems for strongly mixing stationary random measures. Stochastic Process. Appl. 36 231-243.

Leadbetter, M. R., Lindgren, G. and Rootzén, H. (1983). Extremes and Related Properties of Random Sequences and Processes. Springer, New York,

LeAdbetter, M. R. and Rootzén, H. (1988). Extremal theory for stochastic processes. Ann. Probab. 16 431-478.

Matthes, K., Kerstan, J. and Mecke, J. (1978). Infinitely Divisible Point Processes. Wiley, New York.

MORI, T. (1977). Limit distributions of two-dimensional point processes generated by strong mixing sequences. Yokohama Math. J . 25 155-168. 
O'Brien, G., Torfs, P. and VervaAt, W. (1990). Stationary self-similar extremal processes. Probab. Theory Related Fields 87 97-119.

Resnick, S. I. (1987). Point Processes, Regular Variation and Weak Convergence. Springer, New York.

RootzÉn, H. (1978). Extremes of moving averages of stable processes. Ann. Probab. 6 847-869.

Department of Statistics

TEXAS A \& M UNIVERSITY

College Station, TeXas 77843

E-MAIL: thsing@stat.tamu.edu
DePARTMENT OF STATistics

UNIVERSITY OF NORTH CAROLINA

CB 3260, PHILliPS HALL

Chapel Hill, North Carolina 27599-3260

E-MAIL: mrl@stat.unc.edu 